\title{
Theoretical analysis of contributions of self-phase modulation and group-velocity dispersion to femtosecond pulse generation in passive mode-locked dye lasers
}

\author{
A. Penzkofer, M. Wittmann, W. Bäumler, and V. Petrov
}

\begin{abstract}
The influence of self-phase modulation (SPM) and group-velocity dispersion (GVD) on pulse development in a passive mode-locked dye laser is investigated numerically by using fast Fourier transformations. The situation of positive SPM is considered. Four regions of laser performance may be distinguished: (i) In the positive GVD region pulse broadening by GVD must be balanced by the pulse-shortening effect of the saturable absorber. (ii) In a region around zero GVD the laser is periodically self-quenching and the temporal and spectral pulse shapes change periodically similar to higher-order solitons. (iii) It follows a negative GVD region where stable pulses of smooth temporal and spectral shapes are generated similar to fundamental solitons. In this region the pulse duration is practically independent of the saturable absorber concentration, and the saturable absorber is needed mainly for background suppression. (iv) Further increasing the negative GVD, pulse broadening must be balanced by the saturable absorber pulse shortening.

Key words: Self-phase modulation, group-velocity dispersion, femtosecond pulse generation, passive mode locking. spectral filtering, solitonlike pulse propagation.
\end{abstract}

\section{Introduction}

Femtosecond pulses in dye lasers ${ }^{1-3}$ and solid-state lasers (color centers, ${ }^{4} \mathrm{Nd}$ :glass, ${ }^{5,6}$ Ti:sapphire ${ }^{7-14}$ are generated by various mode-locking techniques: saturable absorber mode locking, ${ }^{1-3,9,15-17}$ hybrid synchronous pumping and passive mode locking, 1,3,15,17,18 Kerr lens mode locking, ${ }^{19,20}$ synchronous pumping (gain modulation) and self-phase modulation (SPM)group-velocity dispersion (GVD) balanced operation, ${ }^{12}$ acousto-optic modulation (active loss modulation) and SPM-GVD balanced operation, ${ }^{14}$ additivepulse mode locking or coupled-cavity mode locking. ${ }^{4-6,11,13,21-24}$ For stable short-pulse generation it is crucial to compensate ${ }^{25}$ the frequency chirp that is due to SPM ${ }^{26}$ by the time chirp that is due to GVD. ${ }^{27}$ Generally the GVD is adjusted by using a prism pair or a prism quadruple in the laser resonator. ${ }^{25,28}$ Theoretical studies of the pulse development in pas-

The authors are with Naturwissenschaftliche Fakultät IIPhysik, Universität Regensburg, D-W-8400 Regensburg, Germany.

Received 26 March 1992

0003-6935/92/337067-16\$05.00/0.

- 1992 Optical Society of America. sive mode-locked lasers incorporating GVD and SPM are given in Refs. 1 and 29-37.

In this paper we analyze numerically the influence of SPM and GVD on the pulse propagation of a passive mode-locked dye laser. The parameters used belong to Rhodamine-6G as the gain medium and to DODCI (3,3'-diethyloxadicarbocyanine iodide) as the saturable absorber. The pulse amplification in the gain medium and the pulse absorption in the loss medium are included in the analysis. ${ }^{38,39}$ An instantaneous response of the nonlinear refractive index on the pulse intensity is assumed for SPM and the corresponding frequency chirp (optical Kerr effect). ${ }^{26.40}$ Refractive-index changes that are due to inhomogeneous gain medium depletion and inhomogeneous saturable absorber bleaching are small on a subpicosecond time scale ${ }^{39}$ and are neglected (for inclusion in a pure homogeneous broadening off-resonance excitation model see Refs. 1, 17, and 30). The GVD is approximated by a constant time chirp (linear dependence of transit time on frequency). ${ }^{27}$

In Section 2 the laser model and the equation system are presented. The equations are solved numerically by employing fast Fourier transformations to alternate between temporal description (pulse amplification, pulse absorption, SPM) and spectral 
description (GVD, spectral filtering). The results of the numerical simulations are given in Section 3, where first the effects of GVD and SPM in a single passage through the laser are studied and then the effects of GVD, SPM, saturable absorption, amplification depletion, and spectral filtering on the pulse development are discussed. Positive self-phase modulated pulses are considered. ${ }^{25,41,42}$ The results apply as well to negative self-phase-modulated pulses if the sign of the GVD is changed. In Section 4 the simulation results are compared with experimental results that we obtained recently. ${ }^{42}$

\section{Theoretical Model}

A passive mode-locked femtosecond dye laser oscillator generally consists of resonator mirrors, a gain jet, a loss jet, and a prism arrangement for GVD adjustment. The SPM occurs in the gain jet and the loss jet. The GVD takes place in the gain jet and loss jet, the mirrors, and the prisms. A spectral filtering (narrowing) is caused by the mirrors and the gain medium. In our analysis we separate the various effects in a split-step oscillator model as shown in Fig. 1(a). ${ }^{33.34}$ We do not consider the transient buildup process of the femtosecond pulses by starting with the spontaneous emission ${ }^{38,39}$ but start the calculations with the propagation of a femtosecond pulse (initial parameters are given in Table 1).

The saturable absorption behavior of the modelocking dye is described by an isotropic three-level model [see Fig. 1(b)]. The equations for the level populations $N_{i, A}$ and the laser intensity $I_{L, A}$ read as follows:

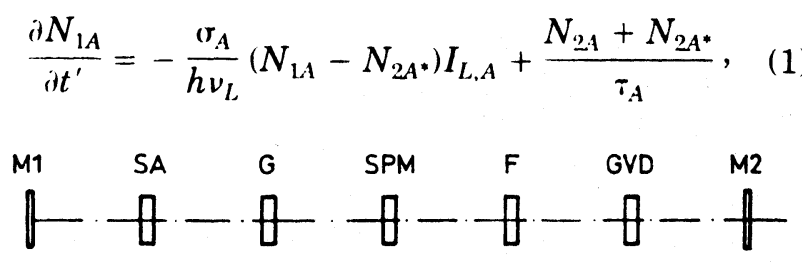

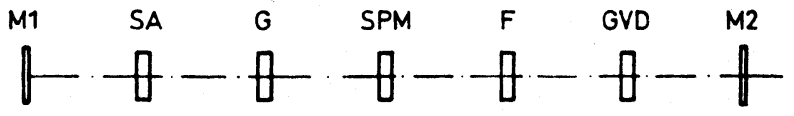

(a)

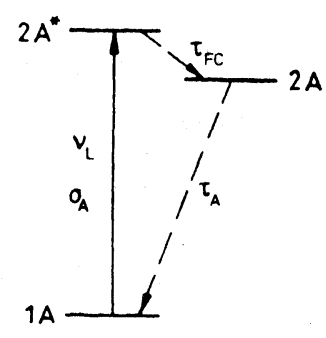

(b)

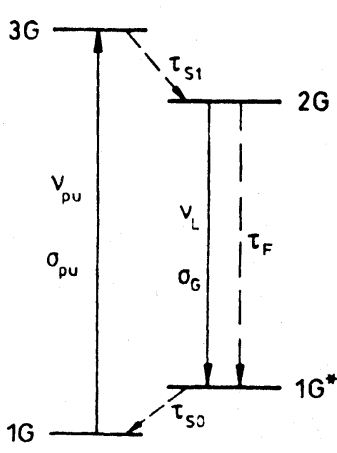

(c)
Fig. 1. (a) Schematic arrangement of a femtosecond laser: M1, front mirror: M2, output mirror (reflectivity $R_{\text {sut }}$ ); SA. saturable absorber jet; $G$, gain jet; SPM, source of self-phase modulation; $F$, source of spectral filtering (spectral narrowing); GVD. source of rroup-velocity dispersion. (b) Three-level model of a saturable absorber. (c) Four-level model of a gain medium.
Table 1. Laser Parameters Applied to Simulations

\begin{tabular}{|c|c|c|}
\hline Parameter & Value & Reference \\
\hline \multicolumn{3}{|l|}{ Laser } \\
\hline$R_{\text {out }}$ & 0.97 & \\
\hline$l_{A}$ & $35 \mu \mathrm{m}$ & \\
\hline$l_{G}$ & $250 \mu \mathrm{m}$ & \\
\hline$f$ & 1 & Eq. (6) \\
\hline$\lambda_{L}$ & $620 \mathrm{~nm}$ & \\
\hline$\Delta \nu_{\mathrm{AMP}} / \Delta v_{L, 0}$ & 8 & Spectral filtering \\
\hline \multicolumn{3}{|c|}{ Absorber (DODCI in ethylene glycol) } \\
\hline$\sigma_{A}$ & $5.2 \times 10^{-16}\left(\mathrm{~cm}^{2}\right)$ & 57 \\
\hline$\tau_{A}$ & $1.4 \mathrm{~ns}$ & 58 \\
\hline $\mathrm{FFC}_{\mathrm{FC}}$ & $0.95 \mathrm{ps}$ & 59 \\
\hline$T_{A 0}$ & 0.85 & \\
\hline$I_{\mathrm{SS} . A^{(0)}}$ & $3.08 \times 10^{9} \mathrm{~W} / \mathrm{cm}^{2}$ & Eq. (5) \\
\hline \multicolumn{3}{|c|}{ Gain medium (Rhodamine-6G in ethylene glycol) } \\
\hline$\sigma_{G}$ & $9 \times 10^{-17} \mathrm{~cm}^{2}$ & 39 \\
\hline$i_{F}$ & $4.1 \mathrm{~ns}$ & 39 \\
\hline iso & $4.0 \mathrm{ps}$ & 60 \\
\hline$G_{0}$ & 1.213 & $T_{A 0}^{-1} R_{\text {out }}^{-1}$ \\
\hline \multicolumn{3}{|c|}{ Initial pulse parameters } \\
\hline$\Delta t_{L .0}$ & $200 \mathrm{fs}$ & \\
\hline$د \bar{\nu}_{L .0}$ & $73.6 \mathrm{~cm}^{-1}$ & Gaussian shape \\
\hline 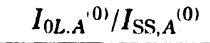 & 3 & \\
\hline
\end{tabular}

$$
\begin{aligned}
\frac{\partial N_{2 A^{*}}}{\partial t^{\prime}} & =\frac{\sigma_{A}}{h v_{L}}\left(N_{1 A}-N_{2 A^{*}}\right) I_{L, A}-\left(\frac{1}{\tau_{\mathrm{FC}}}+\frac{1}{\tau_{A}}\right) N_{2 A^{*}}, \\
\frac{\partial N_{2 A}}{\partial t^{\prime}} & =\frac{N_{2 A^{*}}}{\tau_{\mathrm{FC}}}-\frac{N_{2 A}}{\tau_{A}}, \\
\frac{\partial I_{L A}}{\partial z^{\prime}} & =-\sigma_{A}\left(N_{1 A}-N_{2 A^{*}}\right) I_{L, A} .
\end{aligned}
$$

The transformations $t^{\prime}=t-n_{A} z / c_{0}$ and $z^{\prime}=z$ are used, where $t$ is the time, $n_{A}$ is the refractive index of the absorber solution, $z$ is the distance along the propagation direction, $c_{0}$ is the velocity of light in vacuum, $\nu_{L}$ is the laser frequency, $\sigma_{A}$ is the absorption cross section of the absorber, $\tau_{A}$ is the ground-state absorption recovery time, and $\tau_{\mathrm{FC}}$ is the FranckCondon relaxation time of the excited state.

The initial conditions for each round trip are $N_{1 A}\left(t^{\prime}=-\infty, z\right)=-\ln \left(T_{A 0}\right) /\left(\sigma_{A} l_{A}\right)$ and $N_{2 A}\left(t^{\prime}=-\infty, z\right)$ $=N_{2 A^{*}}\left(t^{\prime}=-\infty, z\right)=0 . T_{A 0}$ is the small-signal double-path transmission and $l_{A}$ is the absorber jet path length.

The absorption saturation by femtosecond pulses may be characterized by the saturation intensity $I_{\mathrm{SS}, A}$ in the slow-saturable-absorber limit $\left(\tau_{A} \gg \Delta t_{L}\right.$, where $\Delta t_{L}$ is the laser pulse duration). $I_{\mathrm{SS}, A}$ is given by ${ }^{38}$

$$
I_{\mathrm{SS}, A}=\frac{h v_{L}}{\sigma_{A} \Delta t_{L}} .
$$

The light pulses may be focused differently to the absorber jet and the gain jet. The input intensity to the gain jet $I_{L, G \text {,in }}$ is related to the output intensity of 
the absorber jet $I_{L, A, \text { out }}$ by

$$
I_{L, G, \text { in }}\left(t^{\prime}\right)=f I_{L, A, \text { out }}\left(t^{\prime}\right),
$$

where $f$ is the focusing parameter $\left(f=d_{A}{ }^{2} / d_{G}{ }^{2}\right.$, where $d_{G}$ is the laser beam diameter in the gain jet and $d_{A}$ is the laser beam diameter in the loss jet).

The pulse amplification and the gain depletion in the gain jet are described by the four-level system of Fig. 1(c). The equation system for the level populations $N_{i, G}$, the femtosecond pulse intensity $I_{L, G}$, and the pump laser intensity $I_{\mathrm{pu}}$ read as follows:

$$
\begin{aligned}
& \frac{\partial N_{1 G}}{\partial t^{\prime}}=-\frac{\sigma_{\mathrm{pu}}}{h \nu_{\mathrm{pu}}}\left(N_{1 G}-N_{3 G}\right) I_{\mathrm{pu}}+\frac{N_{1 G^{*}}}{\tau_{S 0}}, \\
& \frac{\partial N_{3 G}}{\partial t^{\prime}}=\frac{\sigma_{\mathrm{pu}}}{h v_{\mathrm{pu}}}\left(N_{1 G}-N_{3 G}\right) I_{\mathrm{pu}}-\frac{N_{3 G}}{\tau_{S 1}}, \\
& \frac{\partial N_{2 G}}{\partial t^{\prime}}=\frac{N_{3 G}}{\tau_{S 1}}-\frac{N_{2 G}}{\tau_{F}}-\frac{\sigma_{G}}{h \nu_{L}}\left(N_{2 G}-N_{1 G^{*}}\right) I_{L, G}, \\
& \frac{\partial N_{1 G^{*}}}{\partial t^{\prime}}=\frac{\sigma_{G}}{h v_{L}}\left(N_{2 G}-N_{1 G^{*}}\right) I_{L, G}-\frac{N_{1 G^{*}}}{\tau_{S 0}}, \\
& \frac{\partial I_{L, G}}{\partial z^{\prime}}=\sigma_{G}\left(N_{2 G}-N_{1 G^{*}}\right) I_{L, G}, \\
& \frac{\partial I_{\mathrm{pu}}}{\partial z^{\prime}}=-\sigma_{\mathrm{pu}}\left(N_{1 G}-N_{3 G}\right) I_{\mathrm{pu}},
\end{aligned}
$$

where $\sigma_{\mathrm{pu}}$ is the absorption cross section of the gain medium at the pump laser frequency $\nu_{p u}, \tau_{S 1}$ and $\tau_{S 0}$ are the intraband relaxation times in the first excited singlet state $S_{1}$ and in the ground state $S_{0}$, respectively, $\tau_{F}$ is the fluorescence lifetime, and $\sigma_{G}$ is the stimulated emission cross section at the laser frequency $v_{L}$.

The system of Eqs. (7)-(12) may be simplified considerably because of the continuous pumping and the fast intraband relaxation time $\tau_{S 1}$. The fast intraband relaxation $\tau_{S 1} \ll t_{R}$, where $t_{R}$ is the resonator round-trip time, leads to a negligible population of the level $3 G$. Insertion of the steady-state solution of Eq. (8) into Eq. (9) gives

$$
\frac{\partial N_{2 G}}{\partial t^{\prime}}=\frac{\sigma_{\mathrm{pu}}}{h \nu_{\mathrm{pu}}} N_{1 G} I_{\mathrm{pu}}-\frac{N_{2 G}}{\tau_{F}}-\frac{\sigma_{G}}{h \nu_{L}}\left(N_{2 G}-N_{1 G^{*}}\right) I_{L, G} .
$$

The pump laser continuously populates the upper laser level. $I_{\mathrm{pu}}$ is adjusted to keep the energy of the circulating femtosecond pulse constant. The population change of the upper laser caused by the pump laser within the femtosecond pulse passage may be neglected since $\Delta t_{L}$ is short compared with the population accumulation time $\tau_{F}$ of the continuously excited yain dye. Therefore the first term on the right-hand side of Eq. (13) mav be neglected. The relevant equation system for the femtosecond pulse amplifica- tion becomes

$$
\begin{aligned}
& \frac{\partial N_{2 G}}{\partial t^{\prime}}=-\frac{N_{2 G}}{\tau_{F}}-\frac{\sigma_{G}}{h v_{L}}\left(N_{2 G}-N_{1 G^{*}}\right) I_{L, G}, \\
& \frac{\partial N_{1 G^{*}}}{\partial t^{\prime}}=\frac{\sigma_{G}}{h v_{L}}\left(N_{2 G}-N_{1 G^{*}}\right) I_{L, G}-\frac{N_{1 G^{*}}}{\tau_{S 0}}, \\
& \frac{\partial I_{L, G}}{\partial z^{\prime}}=\sigma_{G}\left(N_{2 G}-N_{1 G^{*}}\right) I_{L, G} .
\end{aligned}
$$

In the calculations the initial populations for each round trip are set to

$$
\begin{aligned}
& N_{2 G}\left(t^{\prime}=-\infty, z\right)=\frac{\ln \left(G_{0}\right)}{\sigma_{G} l_{G}}=\frac{\ln \left(T_{\left.A 0^{-1} R_{\text {out }}^{-1}\right)},\right.}{\sigma_{G} l_{G}}, \\
& N_{1 G^{*}}\left(t^{\prime}=-\infty, z\right)=0,
\end{aligned}
$$

i.e., the initial populations for each round trip are such that the small-signal gain $G_{0}=\exp \left(N_{2 G} \sigma_{G} l_{G}\right)$ compensates the small-signal losses $T_{A 0} R_{\text {out }} . l_{G}$ is the path length of the gain jet and $R_{\text {out }}$ is the reflectivity of the output mirror (reflectivity of other resonator mirrors is assumed to be $R=1$ ).

The necessary steady-state pump laser intensity [solution of Eq. (13) without the last term] is approximately given by ${ }^{39}$

$$
I_{\mathrm{pu}} \approx \frac{N_{2 G}\left(t^{\prime}=-\infty, z\right)\left[1-\exp \left(-t_{R} / \tau_{F}\right)\right] h v_{\mathrm{pu}}}{t_{R}\left[1-\exp \left(-\sigma_{\mathrm{pu}} N_{G} l_{G}\right)\right]},
$$

where $N_{G}$ is the total number density of the gain dye molecules. Equations (14)-(18) are used in the simulations of the gain dye amplification in Section 3.

The SPM in the gain jet and loss jet is described in the time space. The relation between intensity $I_{L}$ and electric field amplitude $E_{L}$ is

$$
I_{L}\left(t^{\prime}, z^{\prime}\right)=\frac{n_{0} c_{0} \epsilon_{0}}{2}\left|E_{L}\left(t^{\prime}, z^{\prime}\right)\right|^{2},
$$

with

$$
E_{L}\left(t^{\prime}, z^{\prime}\right)=\left|E_{L}\left(t^{\prime}, z^{\prime}\right)\right| \exp \left[i \phi\left(t^{\prime}, z^{\prime}\right)\right]
$$

where $n_{0}$ is the linear refractive index, $c_{0}$ is the speed of light in vacuum, and $\epsilon_{0}$ is the permittivity of vacuum. At the beginning (number of round trips $j=0$ ), the phase is set to $\phi\left(t^{\prime}, 0\right)=0$.

SPM results from an intensity-dependent change of the refractive index $n .^{26}$ Here we consider only an intensity-dependent instantaneous response of $n$ to $I_{L}$. (response time much faster than pulse duration) as is the case for the electronic optical Kerr effect of the dye solvents. ${ }^{1,26}$ It is

$$
n\left(t^{\prime}\right)=n_{0}+\frac{n_{2}}{2}\left|E_{L}\left(t^{\prime}\right)\right|^{2}=n_{0}+\gamma_{2} I_{L}\left(t^{\prime}\right),
$$

where $\gamma_{2}=n_{2} /\left(n_{0} c_{0} \epsilon_{0}\right), n_{2}$ is the electric field coefficient, and $\gamma_{2}$ is the intensity coefficient of the nonlin- 
ear refractive index. For $n_{2}>0$ we speak of positive SPM. ${ }^{40}$

The SPM changes the temporal phase $\phi_{\text {in }}\left(t^{\prime}\right)$ to

$$
\phi_{\text {out }}\left(t^{\prime}\right)=\phi_{\text {in }}\left(t^{\prime}\right)+\phi_{\text {SPM }}\left(t^{\prime}\right) .
$$

The phase change per resonator round trip (two passages through gain jet and absorber jet) ${ }^{40}$ is

$$
\begin{aligned}
& \phi_{\mathrm{SPM}}\left(t^{\prime}\right) \\
& =\frac{2 \pi v_{L}}{c_{0}} \gamma_{2}\left[2 \int_{0}^{l_{A}} I_{L, A}\left(t^{\prime}, z^{\prime}\right) \mathrm{d} z^{\prime}+2 \int_{0}^{l_{G}} I_{L, G}\left(t^{\prime}, z^{\prime}\right) \mathrm{d} z^{\prime}\right] \\
& \approx \frac{4 \pi v_{L} \gamma_{2}}{c_{0}} I_{L, G}\left(t^{\prime}, l_{G}\right)\left[\frac{l_{A}}{f}+l_{G}\right]
\end{aligned}
$$

The frequency chirp is $v_{c}=-\left(\partial \phi_{\mathrm{SPM}} / \partial t^{\prime}\right) / 2 \pi$, and $\partial v_{c} / \partial t^{\prime}$ is the frequency chirp factor lone speaks of positive frequency chirp or frequency up chirp when $\left.\partial v_{c} / \partial t^{\prime}>0\right)$. The SPM does not change the temporal intensity distribution as is seen by insertion of Eq. (21) into Eq. (20).

The spectral intensity distribution $I_{L}\left(v^{\prime}\right)$ of the laser pulse is found by Fourier transforming $E_{L}\left(t^{\prime}\right)$ to $E_{L}\left(\nu^{\prime}\right)$ by fast Fourier transformation,

$$
\begin{aligned}
E_{L}\left(v^{\prime}\right) & =\int_{-\infty}^{\infty} E_{L}\left(t^{\prime}\right) \exp \left(-i 2 \pi v^{\prime} t^{\prime}\right) \mathrm{d} t^{\prime} \\
& =\left|E_{L}\left(v^{\prime}\right)\right| \exp \left[i \phi\left(v^{\prime}\right)\right]
\end{aligned}
$$

and by using the relation

$$
I\left(v^{\prime}\right)=\frac{c_{0} n_{0} \epsilon_{0}}{4 \pi}\left|E_{L}\left(v^{\prime}\right)\right|^{2}
$$

The spectral intensity transformation between frequency $\nu^{\prime}$ and wave number $\bar{\nu}^{\prime}=v^{\prime} / c_{0}$ is given by

$$
I\left(\tilde{v}^{\prime}\right)=c_{0} I\left(\nu^{\prime}\right) .
$$

The spectral filtering (spectral narrowing) of the laser pulse by the finite spectral gain width and the finite spectral width of the mirror reflectivities is taken into account by multiplying the spectral pulse intensity distribution $I\left(v^{\prime}\right)$ by a Gaussian filter function, i.e., $I_{L, G}$ changes to $I_{L, F}$ according to

$$
I_{L, F}\left(v^{\prime}\right)=I_{L, G}\left(v^{\prime}, l_{G}\right) \exp \left(-v^{\prime 2} / \nu_{A M P}{ }^{2}\right),
$$

where $v_{\mathrm{AMP}}=\Delta v_{\mathrm{AMP}}\left[2(\ln 2)^{1 / 2}\right]^{-1}$ is the effective half of the $1 / e$ spectral amplification width $\left(\Delta v_{\mathrm{AMP}}\right.$ is the FWHM value). The spectral filtering narrows the spectral intensity distribution and thereby broadens the temporal intensity distribution. It causes a temporal pulse structuring when the spectral broadening that is due to SPM is restricted by the spectral filter width (see Figs. 8, 9, and 16 below).

A positive GVD is caused by the normal dispersion of the refractive index in the gain and absorber jets. ${ }^{39}$ The GVD is varied between positive and negative values by proper adjustment of a prism pair or a prism quadruple. The transit time spreading (time chirp) per resonator round trip, $\delta t_{\mathrm{tr}}=t_{\mathrm{tr}}\left(v_{L}=v_{0 L}+v^{\prime}\right)-$ $t_{\mathrm{tr}}\left(v_{0 L}\right)$, is given by $\delta t_{\mathrm{tr}}=\left(\partial t_{\mathrm{tr}} / \partial v\right) v^{\prime}$, where $\partial t_{\mathrm{tr}} / \partial v$ is the time chirp factor. The phase change per round trip, $\phi_{\mathrm{GVD}}(\nu)$, is given by

$$
\begin{aligned}
\phi_{\mathrm{GVD}}\left(\nu^{\prime}\right)-\phi_{\mathrm{GVD}}(0) & =\int_{0}^{\omega^{\prime}} \delta t_{\mathrm{tr}}\left(\omega^{\prime \prime}\right) \mathrm{d} \omega^{\prime \prime} \\
& =2 \pi \int_{0}^{v^{\prime}} \delta t_{\mathrm{tr}}\left(\nu^{\prime \prime}\right) \mathrm{d} \nu^{\prime \prime} \\
& =\frac{1}{2} \omega^{\prime 2} \frac{\partial t_{\mathrm{tr}}}{\partial \omega^{\prime}}=\pi v^{\prime 2} \frac{\partial t_{\mathrm{tr}}}{\partial \nu},
\end{aligned}
$$

where $\omega^{\prime}=2 \pi \nu^{\prime}$ is the angular frequency.

The GVD changes the spectral phase $\phi_{\text {in }}\left(\nu^{\prime}\right)$ of Eq. (25) to

$$
\phi_{\text {out }}\left(\nu^{\prime}\right)=\phi_{\text {in }}\left(\nu^{\prime}\right)+\phi_{\mathrm{GVD}}\left(v^{\prime}\right) .
$$

The transit time spreading that is due to GVD does not change the spectral intensity distribution [see Eqs. (25) and (26)], i.e., $I_{L, \mathrm{GVD}}\left(v^{\prime}\right)=I_{L, F}\left(\nu^{\prime}\right)$.

The temporal intensity distribution after spectral filtering and GVD is obtained by inverse Fourier transformation of $E_{L, \mathrm{GVD}}\left(v^{\prime}\right)=\left|E_{L, G}\left(\nu^{\prime}, l_{G}\right)\right| \exp \left(-v^{\prime 2} \mid\right.$ $\left.2 v_{\mathrm{AMP}}{ }^{2}\right) \exp \left[i \phi_{\text {out }}\left(v^{\prime}\right)\right]$ giving

$$
\begin{aligned}
E_{L, \mathrm{GVD}}\left(t^{\prime}\right) & =\int_{-\infty}^{\infty} E_{L, \mathrm{GVD}}\left(\nu^{\prime}\right) \exp \left(i 2 \pi v^{\prime} t^{\prime}\right) \mathrm{d} v^{\prime}, \\
I_{L, \mathrm{GVD}}\left(t^{\prime}\right) & =\frac{n_{0} c_{0} \epsilon_{0}}{2}\left|E_{L, \mathrm{GVD}}\left(t^{\prime}\right)\right|^{2} .
\end{aligned}
$$

The linear time chirp increases the duration of nonself-phase-modulated pulses but does not cause a temporal modulation. ${ }^{40}$ The laser intensity that returns to the loss medium for the next, $(j+1)$-th, resonator round trip is

$$
I_{L, A}{ }^{(j+1)}\left(t^{\prime}, 0\right)=I_{L, G^{(j)}}\left(t^{\prime}\right) R_{\text {out }} / f .
$$

After each round trip the laser intensity $I_{L, A}$ is corrected for equal pulse energy, i.e.,

$$
\begin{aligned}
I_{L . A^{(j+1)}}\left(t^{\prime}, 0\right) & =\frac{\int I_{L, A^{(j)}\left(t^{\prime}, 0\right) \mathrm{d} t^{\prime}}}{\int I_{L, A^{(j+1)}}\left(t^{\prime}, 0\right) \mathrm{d} t^{\prime}} I_{L, A}{ }^{(j+1)}\left(t^{\prime}, 0\right) \\
& =\kappa I_{L, A}{ }^{(j+1)}\left(t^{\prime}, 0\right),
\end{aligned}
$$

where $\kappa$ is the intensity correction factor. The pump laser intensity $I_{\mathrm{pu}}$ has to be adjusted to give a roundtrip gain of $G=\kappa G_{0}$. In the gain dynamics calculations [Eqs. (14)-(18)] the gain $G_{0}$ was used for simplicity. In real experiments only a temporally constant pump intensity value and therefore a temporally constant $\kappa$ value can be adjusted. Whenever the $\kappa$ values strongly vary with the number of round trips, the real laser output laser intensity strongly changes with the number of round trips and the laser is periodically self-quenched (gain falls below laser threshold and proceeds to above threshold because of the continued pumping; see Figs. 10 and 11 below). 


\section{Simulations}

The parameters used in the numerical calculations apply to a femtosecond Rhodamine-6G-DODCI dye laser operating at $\lambda_{L}=620 \mathrm{~nm}$. The laser parameters are collected in Table 1 . These parameters are used in the calculations except as stated otherwise.

First, the single-passage effects of the SPM and the GVD are presented before multiple round trips in the passive mode-locked laser are discussed. Finally, multiple round-trip effects in the laser without saturable absorber action and gain depletion are analyzed.

\section{A. Single-Passage Effects}

\section{Spectral Broadening by Self-Phase Modulation}

For Gaussian temporal pulse shapes the temporal phase change that is due to SPM is [see Eq. (24)]

$$
\phi_{\mathrm{SPM}}\left(t^{\prime}\right)=\phi_{\mathrm{SPM}, P} \exp \left(-t^{\prime 2} / t_{L .0}{ }^{2}\right),
$$

where $t_{L, 0}=\Delta t_{L, 0} /\left[2(\ln 2)^{1 / 2}\right]$ is half of the 1/e temporal pulse width and $\Delta t_{L, 0}$ is the FWHM pulse duration. In Fig. 2 the normalized spectral pulse width $\Delta v_{L} /$ $\Delta v_{L, 0}$ is plotted versus the peak phase $\Phi_{S P M . P}$, where $\Delta v_{L, 0}$ is the spectral width (FWHM) of the initial non-self-phase-modulated Gaussian pulse of duration $\Delta t_{L, 0}$ and $\phi_{S P M, P}$ is related to the peak normalized frequency chirp $\left(\partial v_{c, P} / \partial t^{\prime}\right) \Delta t_{L, 0}{ }^{2}$ at $t^{\prime}=0$ by $\left(\partial v_{c, P} /\right.$ $\left.\partial t^{\prime}\right) \Delta t_{L, 0}{ }^{2}=(4 \ln 2 / \pi) \phi_{\mathrm{SPM}, P}=0.8825 \phi_{\mathrm{SPM}, P}[\mathrm{Eq}$. (24) is applied in derivation]. The calculations are carried out for zero $\operatorname{GVD}\left(\partial t_{\mathrm{tr}} / \partial v=0\right)$. The kink at $\phi_{\mathrm{SPM}, P} \approx 2.5$ is caused by a shoulder growing beyond half of the pulse height. With rising $\phi_{S P M}, P$ the spectral width grows linearly and the spectrum be-

NORMALIZED PEAK FREQUENCY CHIRP $\left(\partial v_{C P} / \partial t^{\prime}\right) \Delta t_{L, 0}^{2}$

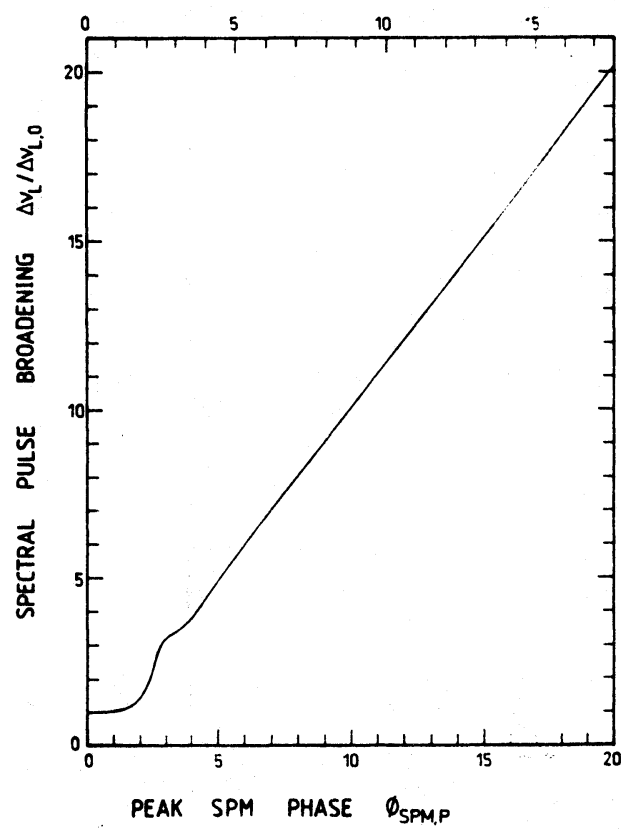

Fig. 2. Dependence of spectral pulse broadening $J \nu_{L} / \Delta \nu_{L, 0}$ on peak temporal phase $\phi_{\text {SPM. }} P$ caused by SPM phase (lower abscissa) or normalized frequency chirp $\left(\partial v_{c, P} / \partial t^{\prime}\right) \Delta t_{L, 0^{2}}$ (upper abscissa). comes modulated. ${ }^{26,40,43}$ The temporal pulse duration and the temporal pulse shape remain unmodified. The spectral broadening is the same for positive SPM $\left(\phi_{\mathrm{SPM}, P}>0\right)$ and negative SPM $\left(\phi_{\mathrm{SPM}, P}<0\right)$.

\section{Temporal Broadening by Group-Velocity Dispersion}

GVD is characterized in the following by the normalized time chirp $\bar{\phi}_{\mathrm{GVD}}$ defined as

$$
\bar{\phi}_{\mathrm{GVD}}=\left(\partial t_{\mathrm{tr}} / \partial \omega\right) \Delta \omega_{L, 0}^{2}=2 \pi\left(\partial t_{\mathrm{tr}} / \partial \nu\right) \Delta \nu_{L, 0}{ }^{2} .
$$

$\bar{\phi}_{\text {GVD }}$ is the effective change of spectral phase within the spectral pulse width that is due to the GVD. The dashed curve in Fig. 3 shows the temporal broadening $\Delta t_{L} / \Delta t_{L, 0}$ versus $\phi_{\mathrm{GVD}}$ in the case of no SPM. The pulse duration rises slowly quadratically with increasing time chirp factor $\left|\partial t_{\mathrm{tr}} / \partial v\right|$. The temporal shape remains smooth (Gaussian); the spectral shape is not modified; and the spectral width $\Delta \nu_{L}$ remains constant.

\section{Combined Self-Phase Modulation and Group-Velocity Dispersion}

The spectral pulse broadening depends only on SPM (is not influenced by the GVD) and is displayed in Fig. 2. The solid curves in Fig. 3 show $\Delta t_{L} / \Delta t_{L, 0}$ versus $\tilde{\phi}_{\mathrm{GVD}}$ for various positive SPM phases $\phi_{\mathrm{SPM}, P}$. In the positive GVD region $\left(\partial t_{\mathrm{tr}} / \partial v>0\right)$ the pulse duration increases with rising $\phi_{\mathrm{SPM}, P}$ because the broadened spectrum enhances the temporal spreading. The temporal shapes are smooth. At $\left(\partial t_{\mathrm{tr}} / \partial v\right)=0$ the pulse duration is independent of $\phi_{\text {SPM }, P}$. In a region

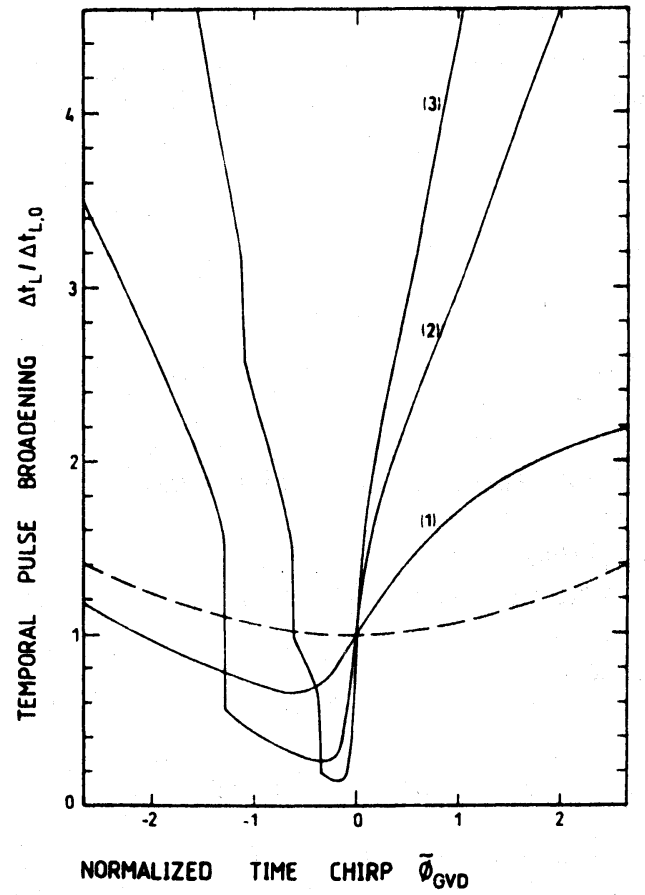

Fig. 3. Dependence of temporal pulse broadening $\Delta t_{L} / \Delta t_{L, 0}$ on normalized time chirp $\dot{\phi}_{\mathrm{GVD}}=2 \pi\left(\partial t_{\mathrm{tr}} / \partial v\right) \Delta v_{L, 0}{ }^{2}$. There is no SPM for the dashed curve. The solid curves belong to $\phi_{\mathrm{SPM} . P}=1(1), 5$ (2), and 10 (3). 
of $-\phi_{\mathrm{SPM}, P^{-1}} \lesssim \tilde{\phi}_{\mathrm{GVD}}<0$ [equivalent to $-1 \leqslant$ $\left.\left(\partial t_{\mathrm{tr}} / \partial v\right)\left(\partial v_{c, P} / \partial t^{\prime}\right)<0\right]$ the pulse duration of selfphase-modulated pulses is shorter than the duration of pulses without SPM. The minimum pulse duration is determined approximately by $\Delta \nu_{L} \Delta t_{L} \approx 0.5$. The positive self-phase-modulated pulses $\left(\partial v_{c, P} / \partial t^{\prime}>\right.$ $0)$ are compressed by the negative time chirp. ${ }^{1,40,43,44}$ When the negative GVD is increased to $\tilde{\phi}_{\mathrm{GVD}} \lesssim$ $-\phi_{\mathrm{SPM}, P^{-1}}$, the negative time chirp overcompensates the positive frequency chirp and the pulse duration broadens.

The vertical steps of the $\Delta t_{L} / \Delta t_{L .0}$ curves in the negative GVD region are caused by the temporal pulse modulation in the overcompensated region. ${ }^{40}$ At each step a temporal spike increases above the half-peak-intensity value.

\section{B. Multiple Round Trips in a Passive Mode-Locked Laser}

First, we consider the situation without SPM, then we study the situation without GVD, followed by investigation of the action of the combined effects of GVD and SPM on the pulse propagation in a passive mode-locked laser. Additionally, the satellite pulse suppression in the laser is discussed.

\section{Group-Velocity Dispersion Effects without Self-Phase Modulation}

In the following analysis no SPM is present $\left(\gamma_{2}=0\right)$. The effect of the GVD depends only on the absolute value of $\dot{\phi}_{\mathrm{GVD}}$. The influences of $\partial t_{\mathrm{tr}} / \partial \nu, \Delta \nu_{\mathrm{AMP}} / \Delta v_{L, 0}$, $I_{0 L, A}{ }^{(0)} / I_{\mathrm{SS}, A^{(0)}}$, and $f=I_{L, G} / I_{L, A}$ are investigated. $I_{0 L . A}{ }^{(0)}$ is the input peak intensity of the laser pulse to the saturable absorber at the beginning of the calculations $(j=0)$, and $I_{\mathrm{SS} . A}{ }^{(0)}$ is the saturation intensity of the slow saturable absorber at the initial pulse duration $\Delta t_{L, 0}$.

The dependence of the normalized pulse duration $\Delta t_{L} / \Delta t_{L, 0}$ on the number of round trips $j$ is shown in Fig. 4. The curves in Fig. 4 belong to different effective spectral phases $\bar{\phi}_{\mathrm{GVD}}$. The pulse duration increases with rising GVD. After a few hundred round trips steady-state pulse durations are approached at which the temporal broadening that is due to GVD is compensated by the saturable absorber action. The spectral pulse development is shown by the solid curves in Fig. 5. With rising GVD the spectral pulse width becomes smaller.

The influence of the finite effective amplification width $\Delta v_{\text {AMP }}$ on the temporal pulse development is illustrated in Fig. 6(a). The normalized pulse durations $\Delta t_{L, 150} / \Delta t_{L, 0}$ after 150 round trips are displayed in Fig. 6 . By reducing the spectral gain width $\Delta v_{\mathrm{AMP}}$, the steady-state pulse durations broaden because of spectral gain narrowing. For large time chirps the steady-state spectral pulse width gets small and the steady-state pulse duration becomes readily independent of $\Delta v_{\text {AMP }}$.

The dependence of the pulse development on the normalized initial peak pulse intensity $I_{0 L, A^{(0)} / I_{\mathrm{SS}, A^{(0)}}}$ is displayed in Fig. 6(c). There is an optimum

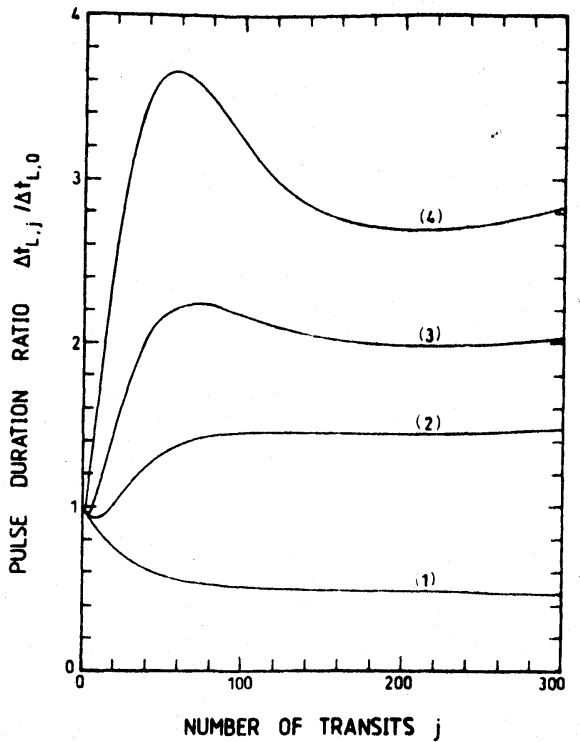

Fig. 4. Dependence of pulse duration $\Delta t_{L}$ on the number of resonator round trips for various GVD's without SPM. The normalized time chirps are $\tilde{\phi}_{\mathrm{GVD}}=0(1), 0.104(2), 0.208(3)$, and 0.416 (4). The parameters of Table 1 apply.

intensity of $I_{0 L, A}{ }^{(0)} / I_{\mathrm{SS}, A^{(0)}} \approx 3$ for the generation of the shortest steady-state pulses. At this intensity value the pulse-shortening action of the saturable absorber is most active. ${ }^{38,39}$

The influence of gain depletion on the steady-state pulse duration is indicated in Fig. 6(b), where $f=$ $I_{L, G} / I_{L . A}$ is varied. The steady-state shortest pulse

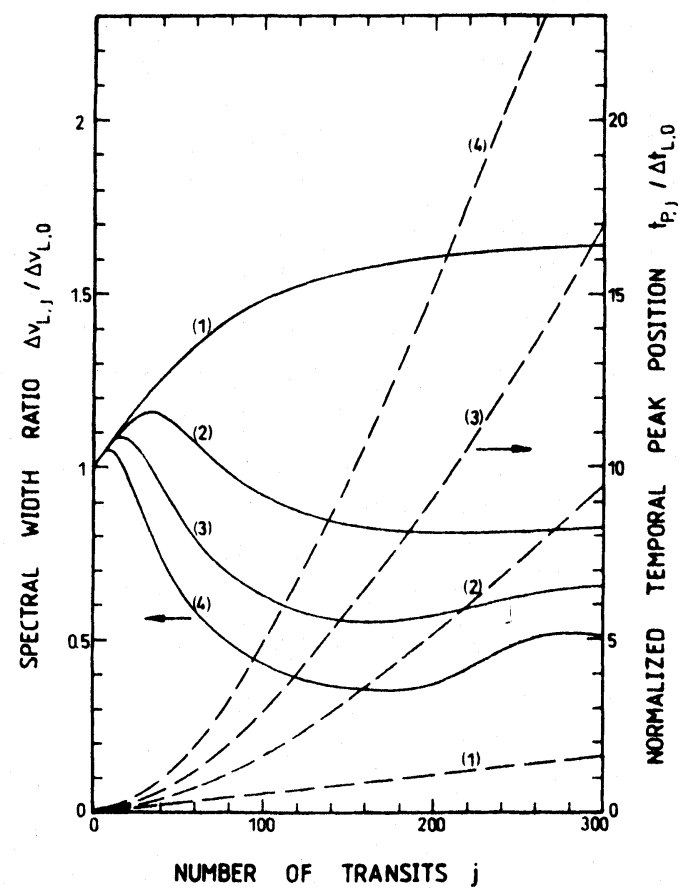

Fig. 5. Dependence of spectral pulse width $\Delta v_{L}$ (solid curves) and of temporal peak pulse position $t_{P}$ (dashed curves) on the number of round trips for various GVD's without SPM. The curves apply to the same parameters as in Fig. 4. 

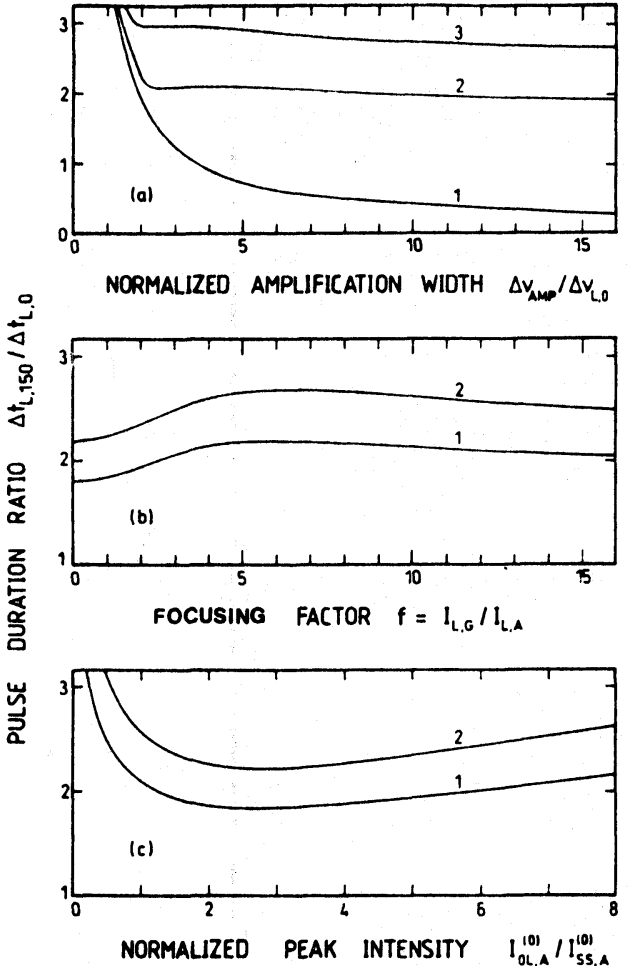

Fig. 6. Dependence of pulse duration $\Delta t_{L}$ in a passive mode-locked laser after 150 round trips on (a) amplification width $\Delta \nu_{\mathrm{AMP}}$; (b) intensity focusing factor $f$; and (c) initial peak laser intensity

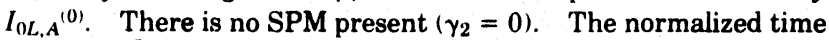
chirp is $\bar{\phi}_{\mathrm{GVD}}=0$ (curves 1 ), 0.208 (curves 2), and 0.416 (curve 3). For (b) and (c) the spectral narrowing is given by $\Delta \nu_{\mathrm{AMP}} / \Delta \nu_{L, 0}=2$; otherwise the parameters of Table 1 apply.

durations are obtained for $f \rightarrow 0$ where no gain depletion occurs. The steady-state pulse durations are longest for $f \approx 7$. The pulse-broadening action results because the leading edge of the pulses is predominantly amplified. ${ }^{39}$ It should be noted that for $f \geq 10\left(I_{L, G} \sigma_{G} / I_{L, A} \sigma_{A} \geq 2\right)$ the ability of background suppression is lost and therefore no selfstarting femtosecond pulse generation (starting from spontaneous emission) is possible. 39,45

The shift of the temporal peak position $t_{P}$ of the pulses as a function of the number of round trips $j$ is shown by the dashed curves in Fig. 5. The shift to longer times is caused by the slow absorption recovery time $\tau_{A}$ of the saturable absorber $\left(\tau_{A} \gg \Delta t_{L}\right)$ that predominantly attenuates the leading edge of the pulse. The temporal shift of the peak pulse position increases with increasing GVD because of the rising pulse duration with rising GVD.

\section{Self-Phase Modulation Effects without Group-Velocity Dispersion}

In the following the passive mode-locked laser is operated under a zero GVD condition by proper adjustment of the intracavity prism arrangement.

The situation without spectral filtering $\left(\Delta v_{\mathrm{AMP}} /\right.$ $\left.\Delta \nu_{1,0} \rightarrow \infty\right)$ is illustrated by the dashed curves in Figs. 7 and 8 . For $\gamma_{2}=0$ and $\partial t_{\mathrm{tr}} / \partial \nu=0$ (dashed curves 1) the pulses are continuously shortened by the saturable absorber action and the spectral width of the bandwidth-limited pulses broadens correspondingly. For $\Delta \nu_{\mathrm{AMP}} \rightarrow \infty$ and $\gamma_{2} \neq 0$ (SPM) the effect of SPM is accumulative and the spectra are continuously broadened and modulated (dashed curve 2 in Fig. 7). The temporal pulse-shortening action of the saturable absorber is not influenced by the SPM [dashed curve 2 for $\gamma_{2}=2 \times 10^{-19} \mathrm{~m}^{2} \mathrm{~W}^{-1}$ coincides with dashed curve 1 for $\gamma_{2}=0$ in Fig. 8(a)]. The shift of the temporal peak position with the number of transits is shown by the dashed curves in Fig. 8(b).

A spectral filtering occurs in the laser by the finite spectral gain width of the active medium and the finite spectral width of the mirror reflectivitie;. The solid curves in Figs. 7 and 8 belong to $\Delta v_{\text {AMP }} / \Delta v_{L .0}=8$. The spectral broadening is limited by the finite effective amplification width $\Delta \nu_{\text {AMP }}$ (solid curves in Fig. 7). For $\gamma_{2} \neq 0$ (SPM) the spectrum is modulated. Abrupt changes of the spectral halfwidth occur whenever spectral spikes rise beyond or decrease below half of the spectral peak value (solid curve 2 in Fig. 7). The restriction of spectral broadening leads to a temporal modulation of the pulse shape and to a periodic abrupt change of the pulse duration [solid curve 2 in Fig. 8(a)]. In regions of short duration a single spike is above the half-peak-height value. In regions of long pulse duration there are 2 or 3 spikes above the half-peak-height value. The spectral and temporal pulse shapes in a region of minimum pulse duration $(j=149)$ and of maximum pulse duration $(j=164)$ are shown in Fig. 9. The parameters are

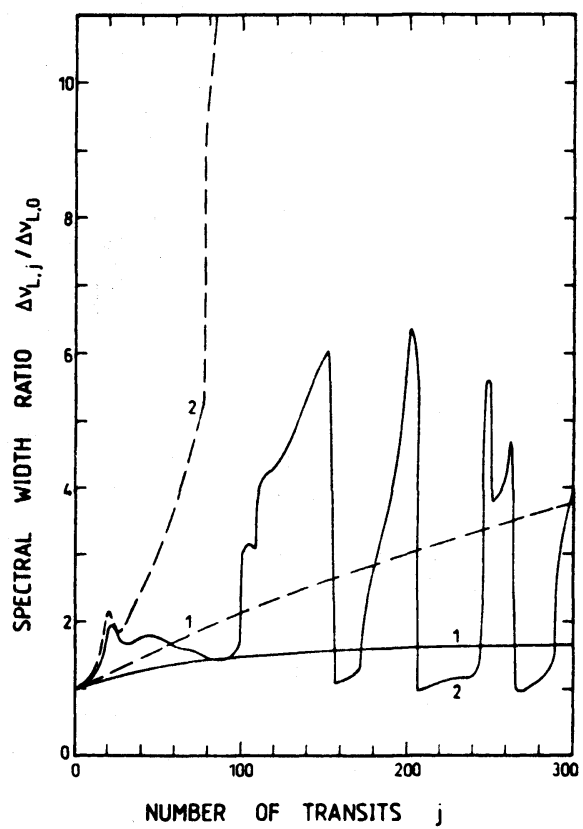

Fig. 7. Dependence of spectral pulse width on the number of resonator round trips in the case of zero GVD for the passive mode-locked laser: dashed curves, $\Delta v_{\text {AMP }} \rightarrow \infty$ (no spectral filtering); solid curves, $\Delta \nu_{\mathrm{AMP}} / \Delta \nu_{l, 0}=8$; curves $1, \gamma_{2}=0$ (no SPM); curves $2, \gamma_{2}=2 \times 10^{-19} \mathrm{~m}^{2} \mathrm{~W}^{-1}$. The other parameters are given in Table 1. 


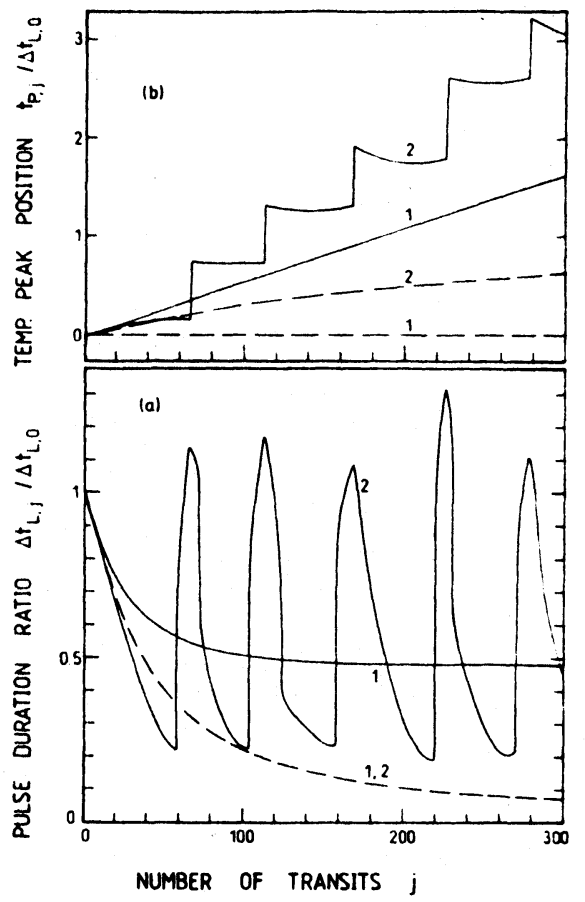

Fig. 8. Dependence of (a) pulse duration $\Delta t_{l}$ and (b) temporal peak position $t_{P}$ on the number of resonator round trips in the case of zero GVD for the passive mode-locked laser. The curves represent the same parameters as in Fig. 7.
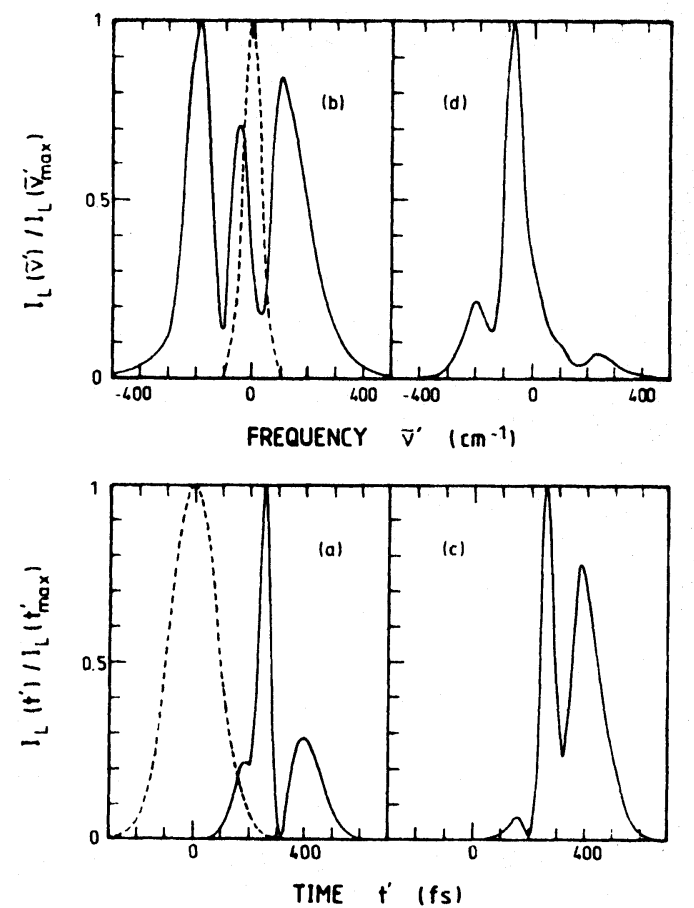

Fig. 9. (a), (c) Temporal and (b), (d) spectral pulse shapes of self-phase-modulated pulses without GVD in a passive modelocked laser (laser operation region $\mathrm{B}$ ). The parameters are the same as for the solid curves 2 in Figs. 7 and $8\left(\gamma_{2}=2 \times 10^{-19} \mathrm{~m}^{2}\right.$ $\mathrm{W}^{1}, \partial t_{\mathrm{tr}} / \partial \nu=0, \Delta \nu_{\mathrm{AMP}} / \Delta v_{l, 0}=8$ ). The number of transits are $j=$ 149 (a), (b) and 164 (c), (d). The dashed curves show the initial pulse shapes. the same as for the solid curves 2 in Figs. 7 and $8\left(\gamma_{2}=\right.$ $2 \times 10^{-19} \mathrm{~m}^{2} \mathrm{~W}^{-1}, \partial t_{\mathrm{tr}} / \partial v=0$ ). The shift of the temporal peak position with the number of transits is displayed by the solid curves in Fig. 8(b).

Curve (B1) in Fig. 10(a) shows the variation of the intensity correction factor $\kappa$ for constant pulse energy along the number of round trips $j$ for $\gamma_{2}=2 \times 10^{-19}$ $\mathrm{m}^{2} \mathrm{~W}^{-1}, \partial t_{\mathrm{tr}} / \partial \nu=0$, and $\Delta \nu_{\mathrm{AMP}} / \Delta \nu_{\mathrm{L}, 0}=8$. The parameters are the same as for the solid curves 2 in Figs. 7 and 8 . In real passive mode-locked lasers only constant or slowly varying $\kappa(j)$ can be realized by the pump laser. A situation of strongly varying $\kappa(j)$ as displayed by curve (B1) in Fig. 10(a) results in a periodically self-quenched laser action. ${ }^{42}$ As the SPM broadens the pulse spectra, the pulse amplification at constant pump rate reduces and the laser falls below threshold (net gain per round trip is $<1$, energy of circulating pulse decreases). Because of the continuous pumping, the laser action and the femtosecond pulse generation restart periodically. The real situation of a constant pump rate is illustrated in Figs. 11(a) and 11(b) where the normalized output pulse energy $W_{L, j} / W_{L, 0}$ and the spectral width ratio $\Delta v_{L 10, j} / \Delta v_{L, 0}$ are displayed versus the number of transits $j$ for $\partial t_{\mathrm{tr}} / \partial v=0, \Delta v_{\mathrm{AMP}} / \Delta v_{L .0}=8, T_{A 0}=0.85$, and $\kappa_{j}=\kappa=1.12 . \Delta \nu_{L 10, j}$ is the full spectral width of the pulse spectrum at one tenth of the peak height for the $j$ th transit.

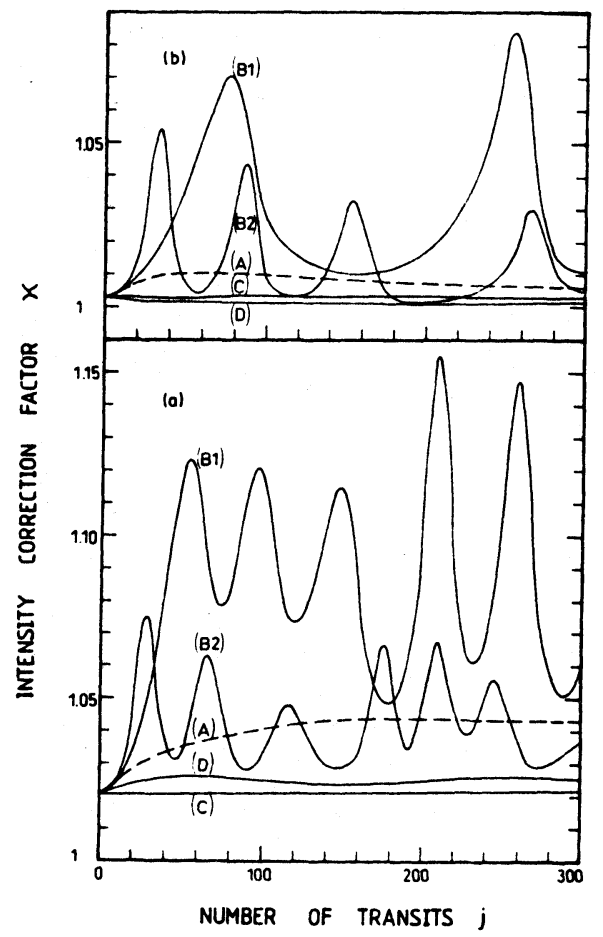

Fig. 10. Intensity correction factor $\kappa$ for conservation of pulse energy in multiple transits for (a) passive mode locking (data of Table 1) and (b) without passive mode locking ( $T_{A 0}=1, G_{0}=$ 1). The curves apply to $\gamma_{2}=2 \times 10^{-19} \mathrm{~m}^{2} \mathrm{~W}^{-1}$ and $\Delta \nu_{\mathrm{AMP}} / \Delta \nu_{L .0}=$ 8. The normalized time chirps are $\bar{\phi}_{\mathrm{G} V \mathrm{~V}}=0.052(\mathrm{~A}), 0$ (B1), -0.052 (B2), -0.208 (C), and -0.831 (D). 


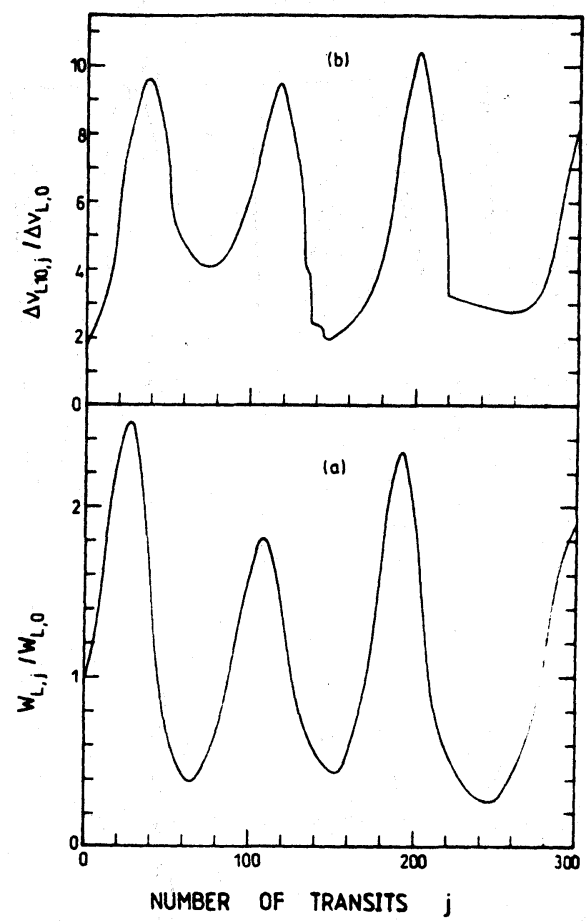

Fig. 11. Variation of (a) normalized pulse energy $W_{L . j} / W_{L, 0}$ and (b) normalized spectral width $\Delta v_{L 10, j} / \Delta v_{L, 0}$ versus the number of round trips $j$ for constant pump laser excitation. $\gamma_{2}=2 \times 10^{-19}$ $\mathrm{m}^{2} \mathrm{~W}^{-1}, \Delta v_{\mathrm{AMP}} / \Delta v_{L, 0}=8, \partial t_{\mathrm{tr}} / \partial \nu=0$, and $\kappa=$ const $=1.12 ;$ the other parameters are given in Table $1 . \Delta v_{L 10}$ is the full spectral width at one-tenth pulse height; $\Delta \nu_{L, 0}$ is the initial spectral half-width (FWHM).

It should be noted that at zero linear time chirp $\partial t_{\mathrm{tr}} / \partial \nu=0$ some GVD remains because of cubic and higher-order terms in the GVD phase $\phi_{\mathrm{GVD}} \cdot{ }^{27.43,44,46,47}$ But these small higher-order terms do not change the periodic self-quenching laser operation (see Figs. 19 and 20 below).

\section{Combined Effects of Self-Phase Modulation and Group-Velocity Dispersion}

The pulse duration ratio $\Delta t_{L, 150} / \Delta t_{L, 0}$ and the spectral width ratio $\Delta v_{L, 150} / \Delta v_{L, 0}$ after 150 resonator round trips (nearly steady-state situation) versus the normalized time chirp $\phi_{G V D}$ are depicted in Figs. 12 and 13, respectively, for various intensity coefficients of the refractive index $\gamma_{2}>0$ [curves (2)-(4), positive SPM] and $\gamma_{2}=0$ [curves (1), no SPM]. The solid curves belong to $\Delta v_{\mathrm{AMP}} / \Delta v_{L, 0}=8$, while the dashed curves belong to $\Delta \nu_{\mathrm{AMP}} \rightarrow \infty$ and $\gamma_{2}=2 \times 10^{-19} \mathrm{~m}^{2} \mathrm{WW}^{-1}$.

In the positive GVD region $\left(\partial t_{\mathrm{tr}} / \partial \nu>0\right)$ the pulse duration rises quickly with increasing time chirp coefficient $\partial t_{\mathrm{tr}} / \partial \nu$. The positive SPM $\left(\gamma_{2}>0\right)$ enhances the temporal pulse spreading. The strong temporal broadening with rising $\partial t_{\mathrm{tr}} / \partial \nu$ reduces the spectral broadening by SPM so that the steady-state spectral width reduces with increasing $i t_{\mathrm{rr}} / \partial \nu$. A steady-state laser operation is achieved by the saturable absorber pulse-shortening action that counteracts the pulse-broadening action of the positive GVD. The temporal and spectral pulse shapes are smooth.

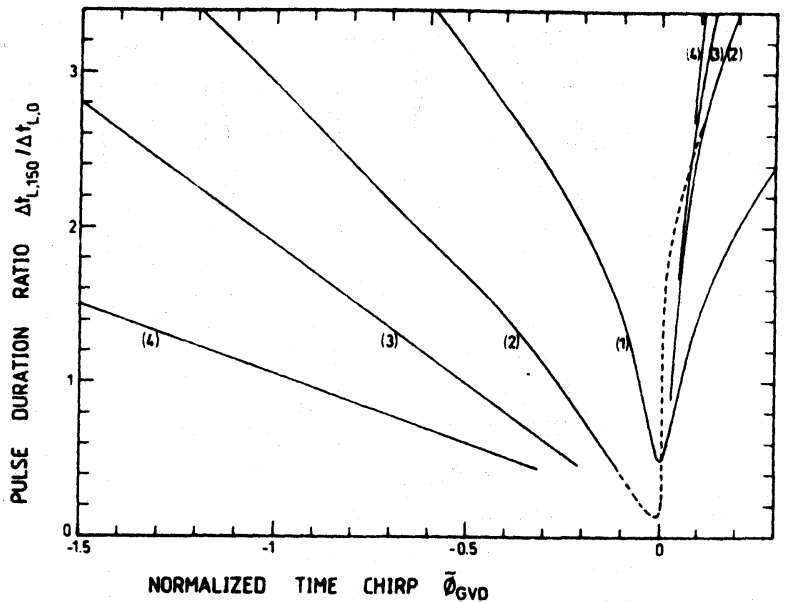

Fig. 12. Dependence of pulse duration on time chirp in a passive mode-locked laser. The pulse duration $\Delta t_{L}$ after $j=150$ transits is displayed for various nonlinear refractive-index coefficients $\gamma_{2}=0$ (1), $2 \times 10^{-19} \mathrm{~m}^{2} \mathrm{~W}^{-1}(2), 4 \times 10^{-19} \mathrm{~m}^{2} \mathrm{~W}^{-1}(3)$, and $8 \times 10^{-19} \mathrm{~m}^{2}$ $\mathrm{W}^{-1}$ (4). The solid curves apply to $\Delta v_{\mathrm{AMP}} / \Delta v_{L, 0}=8$. In the interrupted region around $\partial t_{\mathrm{tr}} / \partial \nu=0$ the durations change periodically in an abrupt manner (self-quenching laser regime). The dashed curve belongs to $\Delta \nu_{\text {AMP }} \rightarrow \infty$ and $\gamma_{2}=2 \times 10^{-19} \mathrm{~m}^{2} \mathrm{~W}^{-1}$; the other parameters are given in Table 1.

For $\gamma_{2}=2 \times 10^{-19} \mathrm{~m}^{2} \mathrm{~W}^{-1}$ and $\tilde{\phi}_{\mathrm{GVD}}=0.052$ the temporal and spectral pulse shape after $j=300$ round trips are displayed by the solid curves in Figs. 14(a) and $14(\mathrm{~b})$, respectively, and the temporal dependence of the intensity correction factor $\kappa$ is shown by dashed curve (A) in Fig. 10(a). The smooth $\kappa(j)$ dependence indicates a stable steady-state pulse propagation.

In a time chirp region around zero GVD that extends from $\partial t_{\mathrm{tr}} / \partial v<0$ to $\partial t_{\mathrm{tr}} / \partial v>0$, the spectral broadening is limited by the spectral filtering of the finite effective amplification width $\Delta v_{\text {AMP. }}$. The tem-

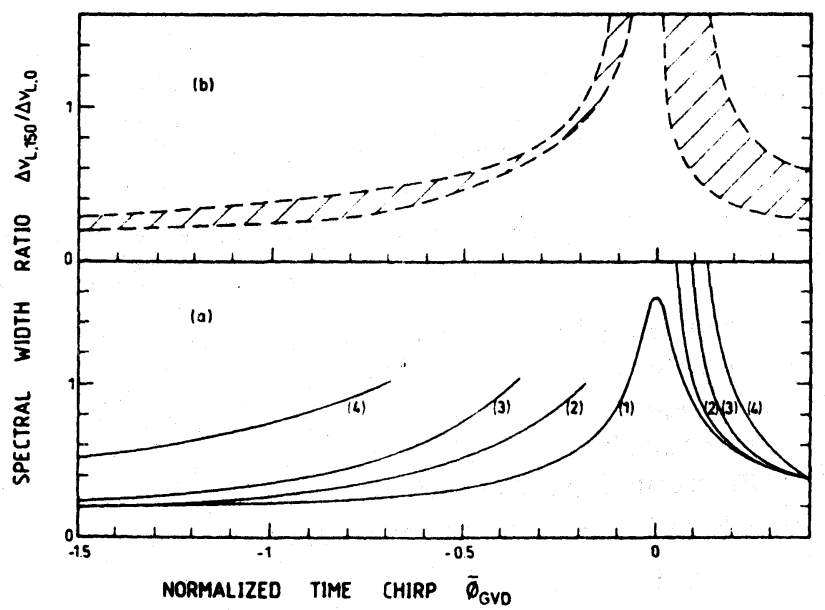

Fig. 13. Dependence of spectral pulse width in a passive modelocked laser on time chirp for various nonlinear refractive-index coefficients $\gamma_{2}$ : (a) $\Delta v_{\mathrm{AMP}} / \Delta \nu_{L .0}=8$ with $\gamma_{2}=0$ (1),2 $2 \times 10^{-19} \mathrm{~m}^{2}$ $\mathrm{W}^{-1}(2), 4 \times 10^{-19} \mathrm{~m}^{2} \mathrm{~W}^{-1}(3)$, and $8 \times 10^{-19} \mathrm{~m}^{2} \mathrm{~W}^{-1}$ (4); (b) $\Delta \nu_{\mathrm{AMP}} \rightarrow \infty$ and $\gamma_{2}=2 \times 10^{-19} \mathrm{~m}^{2} \mathrm{~W}^{-1}$. The hatched region between the dashed borders indicates the region of periodic pulse duration variation within the first 300 round trips. 

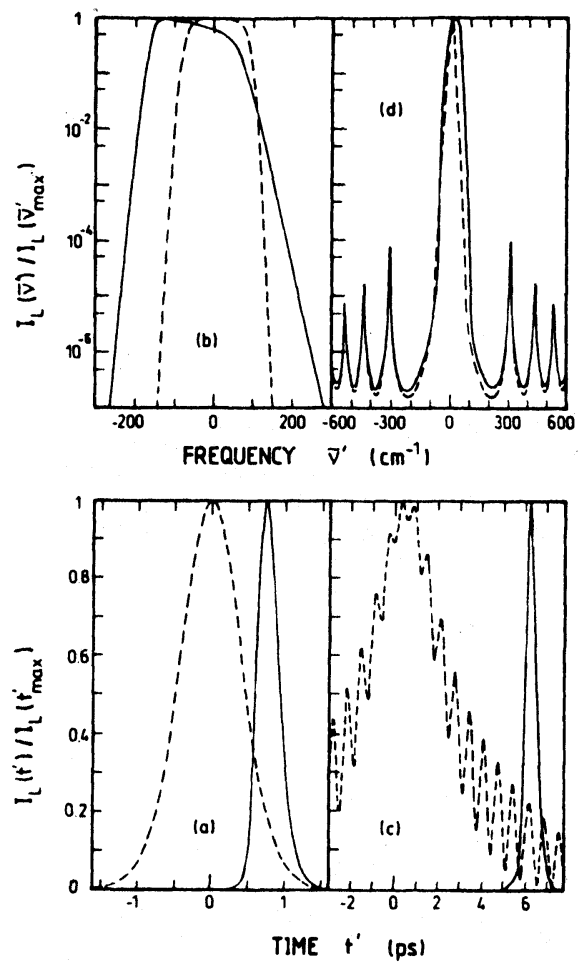

Fig. 14. (a), (c) Temporal and (b), (d) spectral pulse shapes after 300 round trips; $\gamma_{2}=2 \times 10^{-19} \mathrm{~m}^{2} \mathrm{~W}^{-1}$ and $\Delta \nu_{\mathrm{AMP}} / \Delta \nu_{L, 0}=8$ for all curves. The initial pulse shapes are shown by the dashed curves in Figs. 9(a) and 9(b). (a), (b) $\bar{\phi}_{\mathrm{GVD}}=\mathbf{0 . 0 5 2}$ (laser operation region A) with saturable absorber action (solid curves) and without a saturable absorber (dashed curves). (c), (d) $\dot{\phi}_{\mathrm{GVD}}=-0.714$ (laser operation region D) with a saturable absorber (solid curves) and without a saturable absorber (dashed curves).

poral and spectral pulse shapes of the self-phasemodulated pulses are modulated. The spectral and temporal half-widths change periodically in an abrupt manner [similar to solid curves 2 in Figs. 7 and 8(a)]. In this spectral and temporal modulation region the solid curves in Figs. 12 and 13 are interrupted. Solid curve (B2) in Fig. 10(a) shows the modulation of $\kappa(j)$ for $\bar{\phi}_{\mathrm{GVD}}=-0.052$. In the experimental situation the laser action is periodically self-quenching in the interrupted regime because the laser falls below threshold by the spectral broadening of the SPM effect.

In a negative GVD region near the zero GVD position $\partial t_{\mathrm{tr}} / \partial v=0$ beyond the periodically selfquenching region, the steady-state pulse durations are shorter than the initial pulse duration $\Delta t_{L, 0}$. The bandwidth product $\Delta v_{L} \Delta t_{L}$ reduces below the initial value of $\Delta v_{L, 0} \Delta t_{L, 0}=2 \ln (2) / \pi$ (bandwidth-limited Gaussian pulses). A temporal and spectral pulse shape at $\phi_{\mathrm{GVD}}=-0.208$ is shown by the solid curves in Figs. 15(a) and 15(b), respectively, after 300 round trips. Solid curve (C) in Fig. 10(a) displays a nearly constant $\kappa(j)$ for $\tilde{\phi}_{\mathrm{GVD}}=-0.208$.

With increasing negative GVD the pulse durations broaden. This temporal broadening reduces the SPM effect and the spectral halfwidth reduces. For the solid curves the spectral and temporal pulse shapes are smooth. The rise of pulse duration with increas-
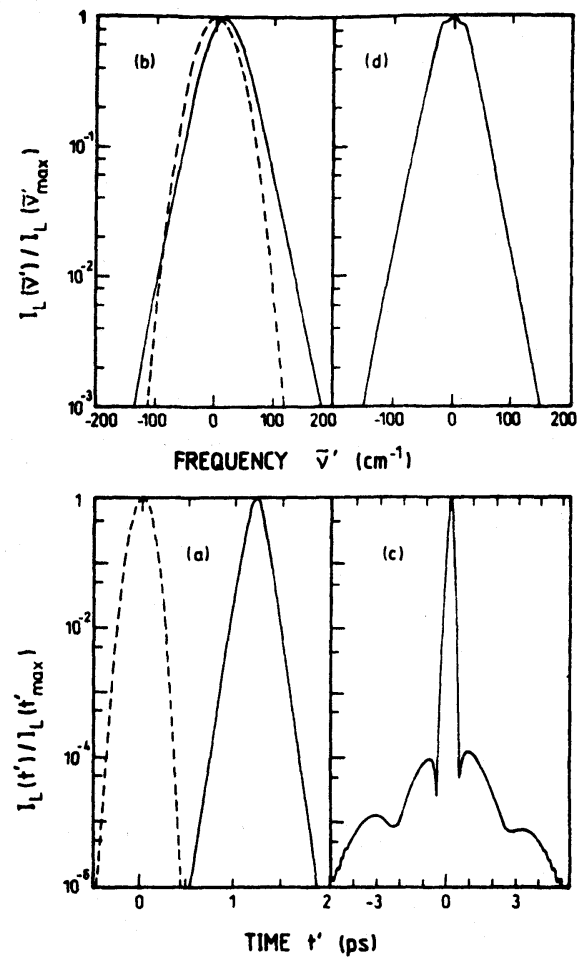

Fig. 15. (a), (c) Temporal and (b), (d) spectral pulse shapes of self-phase-modulated pulses in the negative GVD region after 300 round trips; $\gamma_{2}=2 \times 10^{-19} \mathrm{~m}^{2} \mathrm{~W}^{-1} ; \bar{\phi}_{\mathrm{GVD}}=-0.208$ (stable laser operation region C): (a), (b) passive mode-locked laser; (c), (d) pulse propagation in the resonator without a saturable absorber. The parameters of Table 1 apply otherwise. The dashed curves in (a) and (b) show the initial pulse shapes.

ing negative GVD is much slower than the steep rise of pulse duration with increasing positive GVD. The positive frequency chirp (caused by the positive SPM) and the negative time chirp (negative GVD) combine subtractively and reduce the temporal and spectral broadening. For $\phi_{\mathrm{GVD}}=-0.714$, the temporal and spectral pulse shapes after 300 round trips is shown by the solid curves in Figs. 14(c) and 14(d). The slowly varying curve (D) in Fig. 10(a) shows $k(j)$ for $\bar{\phi}_{\mathrm{GVD}}=-0.831$.

The dashed curves in Figs. 12 and 13(b) are calculated for $\gamma_{2}=2 \times 10^{-19} \mathrm{~m}^{2} \mathrm{~W}^{-1}$ and $\Delta \nu_{\mathrm{AMP}} \rightarrow \infty$. The temporal pulse shapes remain smooth in the region around zero GVD. The spectral shapes are slightly modulated and the spectral half-width changes periodically with the number of round trips. The spectral half-width variation within the first 300 round trips is indicated by the hatched area within the dashed borders. In the region around zero GVD, the spectral broadening continues to increase with the number of round trips (no steady-state value is approached). The intensity correction factor $\kappa(j)$ is constant and independent of $\partial t_{\mathrm{tr}} / \partial \nu(\mathrm{\kappa} \approx 1.017$ for the parameters in Table 1).

\section{Background Suppression}

Saturable absorbers with a fast intraband partial absorption recovery have the potential of background suppression. ${ }^{39,40}$ Here the background suppression 

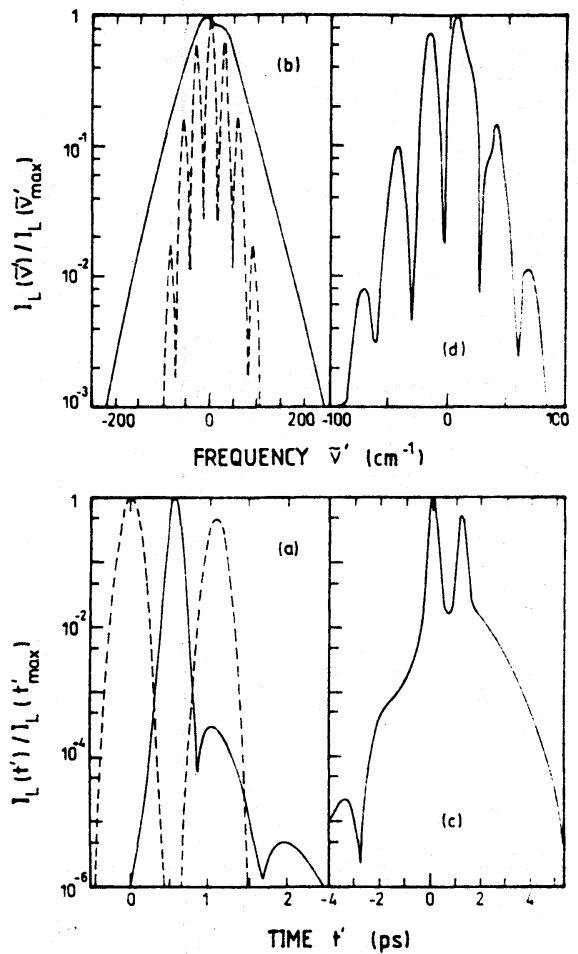

Fig. 16. Main pulse and satellite pulse reshaping in the resonator. The number of round trips is $j=300$. The initial temporal and spectral shapes are shown by the dashed curves in (a) and (b). Otherwise the parameters are the same as in Fig. 15 [for (a) and (b) with a saturable absorber; for (c) and (d) without a saturable absorber].

action is illustrated in Figs. 16(a) and 16(b). The propagation of a main pulse and a satellite pulse is considered. The initial temporal and spectral pulse shapes are shown by the dashed curves. The initial spectrum is modulated by the presence of the satellite pulse. ${ }^{48}$ The SPM and GVD data are selected to be $\gamma_{2}=2 \times 10^{-19} \mathrm{~m}^{2} \mathrm{~W}^{-1}\left[\phi_{\mathrm{SPM}, P^{(0)}}=0.1068\right]$ and $\tilde{\phi}_{\mathrm{GVD}}=$ -0.208 , respectively (region of stable femtosecond pulse generation). The spectral filtering is $\Delta \nu_{\text {AMP }} /$ $\Delta \nu_{L, 0}=8$. The satellite light peak intensity reduces from $I_{\text {sat }, P} / I_{L, P}=0.5$ at $j=0$ to $3 \times 10^{-4}$ at $j=300$. The spectral shape becomes rather smooth because of the satellite pulse suppression.

\section{Multiple Resonator Round Trips without Passive Mode Locking}

The pulse-shortening action of the saturable absorber and the pulse-broadening action of the gain medium ${ }^{39}$ are excluded in this section $\left(T_{A 0}=1, G_{0}=1\right)$ in order to study the pulse-forming effects of GVD and SPM separately. The situations of GVD without SPM, of SPM without GVD, and the combined action of SPM and GVD are considered. The problem of background suppression is discussed.

\section{Group-Velocity Dispersion without Self-Phase Modulation}

The curves 1 in Figs. 17 and 18 show the dependences of the pulse durations $\Delta t_{L, j}$ and of the spectral widths $\Delta v_{L, j}$ on the number of transits $j$, respectively, for

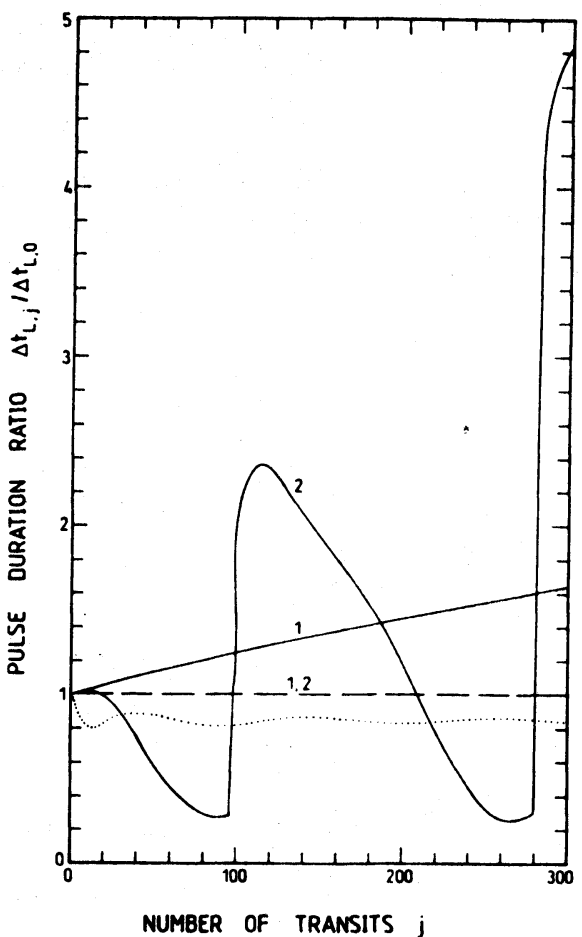

Fig. 17. Development of the pulse duration in multiple transitions through the resonator without a saturable absorber $\left(T_{A 0}=1\right)$ and without active medium action $\left(G_{0}=1\right)$. The dashed curves represent $\lrcorner \nu_{\mathrm{AMP}} \rightarrow \infty$ and zero GVD. The solid curves represent $\Delta \nu_{\mathrm{AMP}} / \Delta v_{L .0}=8$ and zero GVD. Curves $1, \gamma_{2}=0$ (no SPM); curves $2, \gamma_{2}=2 \times 10^{-19} \mathrm{~m}^{2} \mathrm{~W}^{-1}$; the dotted curve represents $\gamma_{2}=2 \times$ $10^{-19} \mathrm{~m}^{2} \mathrm{~W}^{-1}, \bar{\phi}_{\mathrm{GVD}}=-0.208$, and $\Delta v_{\mathrm{AMP}} i \Delta \nu_{L, 0}=8$. The other parameters are taken from Table 1.

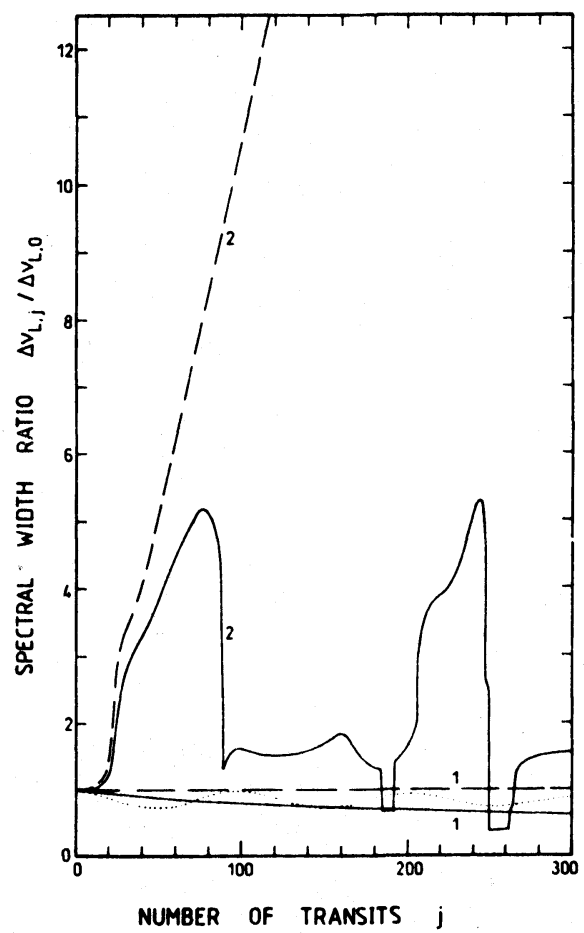

Fig. 18. Development of the spectral pulse width in multiple resonator round trips for $T_{A 0}=1$ and $G_{0}=1$. The curves represent the same parameters as in Fig. 17. 
zero GVD and zero SPM. In the case of spectral filtering (solid curves $1, \Delta \nu_{\mathrm{AMP}} / \Delta \nu_{L, 0}=8$ ) the pulse duration increases gradually and the spectral width narrows gradually. No steady-state pulse duration is approached. Without spectral filtering $\left(\Delta v_{\mathrm{AMP}} \rightarrow\right.$ $\infty$, dashed curves 1 ) the temporal and spectral widths remain constant.

The dependence of the pulse duration on the time chirp after 300 round trips is shown in Fig. 19(a). The pulse duration $\Delta t_{L, 300}$ is shortest for zero GVD. It rises quadratically with $\left|\partial t_{\mathrm{tr}} / \partial \nu\right|$. The solid curve represents $\Delta \nu_{\mathrm{AMP}} / \Delta \nu_{L, 0}=8$ (spectral filtering) and the dashed curve represents $\Delta v_{\mathrm{AMP}} \rightarrow \infty$. For $\partial t_{\mathrm{tr}} / \partial \nu \neq 0$ no steady-state pulse duration is approached because of the continuing pulse spreading by the GVD. The spectral pulse width does not depend on the GVD as is shown in Fig. 20(a).

\section{Self-Phase Modulation without Group-Velocity Dispersion}

The spectral pulse broadening that is due to SPM is illustrated by curves 2 in Fig. 18 . Without spectral filtering (dashed curve 2) the spectral width grows continuously with the number of round trips. The spectra are modulated. The intensity correction factor is $k(j)=1$. A spectral filtering limits the

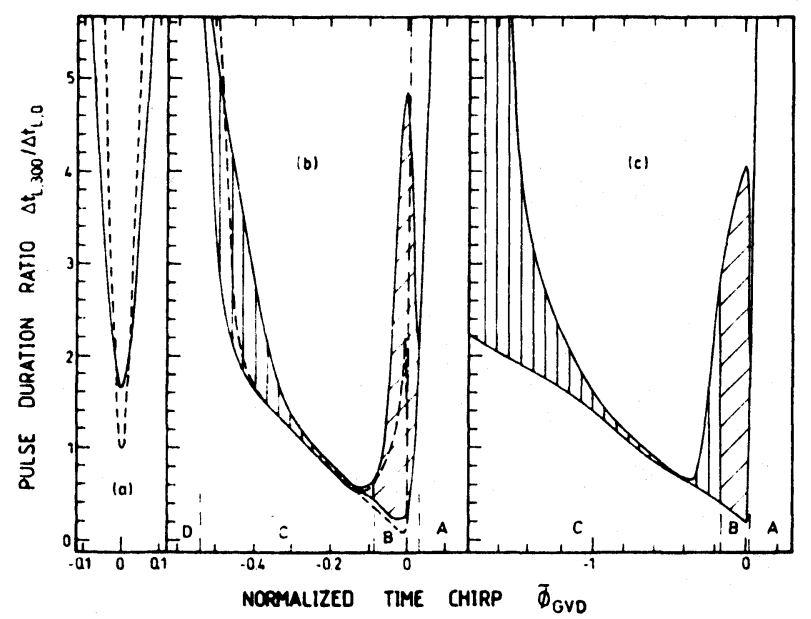

Fig. 19. Dependence of the pulse duration on GVD in the resonator without a saturable absorber and without gain medium action ( $\left.T_{A 0}=1, G_{0}=1\right)$. Otherwise the parameters of Table 1 apply. Solid curves, $\Delta \nu_{\mathrm{AMP}} / \Delta \nu_{l .0}=8$ (spectral filtering); dashed curves, $\Delta v_{\mathrm{AMP}} \rightarrow \infty$. (a) $\gamma_{2}=0$ (no SPM); the curves represent 300 round trips; no steady-state pulse duration is reached for $\partial t_{\mathrm{tr}} / \partial \nu \neq$ 0. (b) $\gamma_{2}=2 \times 10^{-19} \mathrm{~m}^{2} \mathrm{~W}^{-1}$; (c) $\gamma_{2}=8 \times 10^{-19} \mathrm{~W}^{-1}$. Region $\mathrm{A}$, pulse duration after 300 round trips. The duration continues to increase. Region B (periodically self-quenched region), the pulse duration varies periodically within the diagonally hatched region (similar to Fig. 8). For $\Delta v_{\mathrm{AMP}} / \Delta v_{l, 0}=8$ the mean pulse duration continues to increase slowly with the number of transits. Region (: (fundamental solitonlike region), the pulse duration oscillates smoothly within the vertically hatched region (see the dotted curve in Fig. 17). The pulse duration remains within the hatched region with the number of round trips (checked as many as 900 transits). Region $\mathrm{D}$, the pulse duration continues to increase with the number of round trips. spectral broadening (solid curve 2). The spectral shapes are modulated. The spectral widths change abruptly whenever spectral spikes rise above or fall below half of the peak value. The behavior is similar to the passive mode-locked situation (Fig. 7). The intensity correction factor $\kappa$ is modulated as is seen by solid curve (B1) in Fig. 10(b) (periodic self-quenching behavior).

The temporal pulse behavior that is due to SPM is shown by curves 2 in Fig. 17 . Without spectral filtering the pulse duration remains constant, independent of the number of transits. The spectral filtering causes a modulation of the temporal pulse shape. An abrupt rise of the pulse duration occurs whenever spikes grow beyond the half-peak value. The average pulse duration increases with the number of transits (no steady-state value is approached).

\section{Combined Effects of Self-Phase Modulation and Group-Velocity Dispersion}

The temporal and spectral pulse development in the case of SPM and GVD is illustrated in Figs. 19(b) and 19 (c) and 20(b) and 20(c). The temporal and spectral widths after 300 resonator round trips are displayed in the case for which the half-width changes monotonically with the number of transits. Otherwise the hatched regions indicate the variation range within the first 300 round trips. The nonlinear refractive-index coefficients are $\gamma_{2}=2 \times 10^{-19} \mathrm{~m}^{2}$ $\mathrm{W}^{-1}\left[\phi_{\mathrm{SPM}, P^{(0)}}=0.1068\right]$ for Figs. $19(\mathrm{~b})$ and $20(\mathrm{~b})$ and $\gamma_{2}=8 \times 10^{-19} \mathrm{~m}^{2} \mathrm{~W}^{-1}\left[\phi_{\mathrm{SPM}, P^{(0)}}=0.4272\right]$ for Figs. 19 (c) and 20 (c).

Moving from large positive GVD $\left(\partial t_{\mathrm{tr}} / \partial \nu>0\right)$ toward large negative GVD $\left(\partial t_{\mathrm{tr}} / \partial \nu<0\right)$, we can distinguish four different operation regimes (these regions are indicated in Figs. 19 and 20 for the solid curves, i.e., $\Delta \nu_{\mathrm{AMP}} / \Delta v_{L, 0}=8$ ):

(i) In region $\mathrm{A}$ the pulse duration rises strongly with increasing positive time chirp coefficient

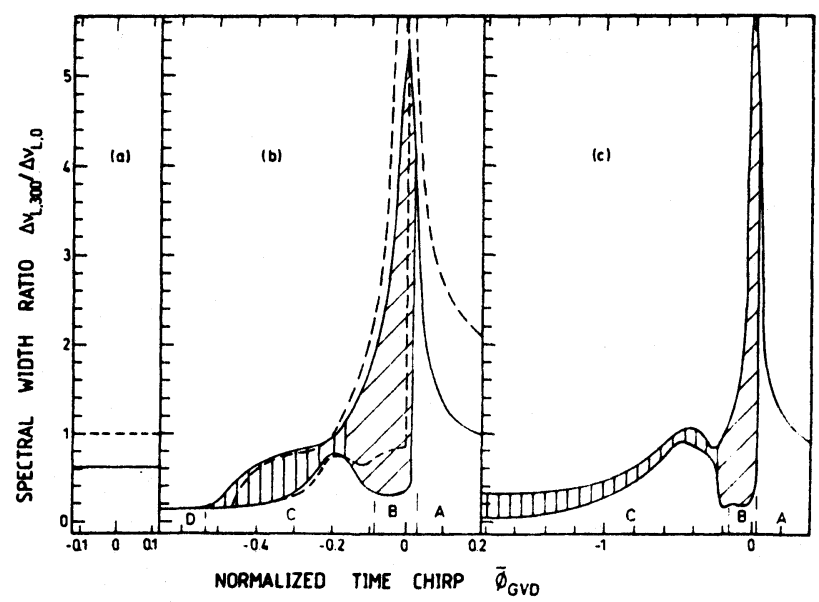

Fig. 20. Dependence of the spectral pulse width on GVD in the resonator without a saturable absorber and without gain medium action. The curves in (a)-(c) represent the same parameters as in Fig. 19. 
$\partial t_{\mathrm{tr}} / \partial v>0$. The temporal broadening is enhanced if no spectral filtering is present [dashed curve in Fig. 19(b)]. The temporal broadening increases continuously with the number of round trips. The spectral widths decrease with rising positive GVD because for longer pulses the effect of SPM is reduced. The spectral width is broader if no spectral filtering is present. The temporal and spectral shapes are smooth as is illustrated by the dashed curves in Figs. $14(\mathrm{a})$ and $14(\mathrm{~b})\left(\gamma_{2}=2 \times 10^{-19} \mathrm{~m}^{2} \mathrm{~W}^{-1}, \bar{\phi}_{\mathrm{GVD}}=0.052\right.$, $\Delta v_{\mathrm{AMP}} / \Delta v_{L, 0}=8$ ). For these data $\kappa(j)$ is shown by dashed curve (A) in Fig. 10(b). Without spectral filtering, i.e., $\Delta \nu_{\mathrm{AMP}} \rightarrow \infty$, it is $\kappa(j)=1$.

(ii) In region $B$ the temporal and spectral shapes are modulated and they change their shape quasiperiodically with the number of transits. The temporal and spectral half-widths change abruptly whenever a spike increases above or decreases below the half-peak value. Within the first 300 round trips, the half-widths vary within the diagonally hatched regions. In the case of spectral filtering (solid curves, $\Delta \nu_{\mathrm{AMP}} / \Delta v_{L, 0}=8$ ) the average temporal half-width continues to increase with the number of round trips. $\kappa(j)$ is modulated as is seen by solid curves (B1) and (B2) in Fig. 10(b) (periodic self-quenching behavior for constant pump pulse excitation). The temporal and spectral pulse development shows some higherorder solitonlike behavior. ${ }^{27,35,37,43,44.46,47,49-52}$ The higher-order solitonlike behavior includes the zero GVD position and extends slightly to the positive GVD region. In the case of no spectral filtering [dashed curves in Figs. 19(b) and 20(b)] the higherorder solitonlike region is restricted to $\partial t_{\mathrm{tr}} / \partial \nu<0$. The soliton order increases toward $\partial t_{\mathrm{tr}} / \partial \nu \rightarrow-0$. The pulse shapes and half-width repeat periodically (true higher-order solitonlike behavior). ${ }^{37}$ The intensity correction factor is $\kappa(j)=1$.

(iii) In region $C$ (in the negative GVD range) a stable pulse propagation occurs. The temporal and spectral pulse widths oscillate around mean values as is seen by the dotted curves in Figs. 17 and 18. The vertically hatched areas in Figs. 19 and 20 show the range of monotonic modulation. There the spectral and temporal shapes are rather smooth. In Fig. 15(c) a temporal pulse shape and in Fig. 15(d) a spectral pulse shape are shown. The temporal and spectral behavior indicates a fundamental solitonlike behavior $\left(N=1\right.$ solitonlike). ${ }^{27,35,37,43,44,49}$ The positive frequency chirp $\partial v_{c} / \partial t$ is compensated (canceled) by the negative time chirp $\partial t_{\mathrm{tr}} / \partial \nu$. In the course of the round trips the pulse duration adjusts in such a way that a compensation of frequency chirp and time chirp occurs. In a certain GVD range the steadystate pulse duration is even shorter than the initial pulse duration. A steady-state temporal and spectral pulse propagation is obtained for both $J \nu_{\mathrm{AMP}} /$ $\Delta v_{L, 0}=8$ (in calculations of as many as 900 round trips the pulse durations and spectral widths re- mained within the hatched region) and $\Delta \nu_{\text {AMP }} \rightarrow \infty$. The steady-state temporal and spectral widths are practically the same for $\Delta v_{\mathrm{AMP}} / \Delta v_{L, 0}=8$ (solid curves) and $\Delta v_{\mathrm{AMP}} \rightarrow \infty$ (dashed curves). $\kappa(j)$ is shown by curve (C) in Fig. 10(b) for $\Delta v_{\mathrm{AMP}} / \Delta v_{L, 0}=8$ and $\bar{\phi}_{\mathrm{GVD}}=$ -0.208 . The extension of the negative GVD range for stable steady-state pulse propagation (region $\mathrm{C}$ ) depends on the strength of the SPM. It does not exist for zero SPM [Figs. 19(a) and 20(a)] and it broadens with increasing positive SPM as is seen in Figs. 19(b) and 19(c) and 20(b) and 20(c).

(iv) In region $\mathrm{D}$ the pulse duration continuously grows with the number of round trips $j$ (no steadystate situation is approached). The temporal broadening increases with rising negative GVD (growing $\left.\left|\partial t_{\mathrm{tr}} / \partial v\right|\right)$. The temporal shape of the long pulses is modulated as is seen by the dashed curve in Fig. 14(c) for $\gamma_{2}=2 \times 10^{-19} \mathrm{~m}^{2} \mathrm{~W}^{-1}, \bar{\phi}_{\mathrm{GVD}}=-0.714$, and $\Delta v_{\mathrm{AMP}} / \Delta v_{L, 0}=8$. The spectral width has a steadystate value that is well below the initial spectral width. For $\gamma=2 \times 10^{-19} \mathrm{~m}^{2} \mathrm{~W}^{-1}, \bar{\phi}_{\mathrm{GVD}}=-0.831$, and $\Delta \nu_{\mathrm{AMP}} / \Delta \nu_{L, 0}=8, \kappa(j)$ is shown by the solid curve (D) in Fig. 10(b). The negative time chirp overcompensates the positive frequency chirp and leads to a continuous temporal pulse broadening. The spectral pulse shape is modulated in the wings [see dashed curve in Fig. 14(d)].

In real passive mode-locked lasers the regions A, B, $\mathrm{C}$, and $\mathrm{D}$ are characterized by the following laser performances: region A gives the positive GVDsaturable absorber balanced regime; region $B$ describes the periodic self-quenching laser regime; region $\mathrm{C}$ is the stable negative GVD balanced laser operation regime (prism balanced laser operation); and region D presents the negative GVD overcompensated saturable absorber balanced regime.

\section{Satellite Pulse Effects}

In Subsection 3.C.3 it was shown that even without saturable absorber action stable steady-state fundamental solitonlike pulse propagation occurs in the negative GVD region $C$ where the negative time chirp compensates the positive frequency chirp. For selfstarting femtosecond lasers a background suppression mechanism is necessary. In the stable pulse propagation region $\mathrm{C}$ we studied the propagation of a main pulse and a satellite pulse. An example is shown in Figs. 16(c) and 16(d). The initial pulses are shown by the dashed curves in Figs. 16(a) and 16(b). No satellite pulse suppression occurred.

For femtosecond lasers that operate in the SPMGVD balanced region C, a passive mode-locking mechanism is necessary for background and satellite pulse suppression such as partial fast saturable absorption (used in Subsection 3.B of this paper ${ }^{42}$ hybrid modelocking, ${ }^{17.18}$ Kerr lens mode locking, ${ }^{19,20}$ synchronous pumping, ${ }^{12}$ active loss modulation, ${ }^{14}$ or additive pulse mode locking. ${ }^{4-6,11,13,21-24}$ 


\section{Comparison with Experiments}

Recently we investigated experimentally the femtosecond pulse generation in a linear passive mode-locked Rhodamine-6G-DODCI dye laser. ${ }^{42}$ The saturable absorber, gain medium, and resonator parameters used in the simulations apply to our experimental situation. The experimental nonlinear refractiveindex coefficient $\gamma_{2}$ is from approximately $2 \times 10^{-20}$ $\mathrm{m}^{2} \mathrm{~W}^{-1}$ to $3 \times 10^{-20} \mathrm{~m}^{2} \mathrm{~W}^{-1}\left(\gamma_{2}\right.$ of the solvent ethylene glycol ${ }^{53,54}$ ). The GVD was varied between $\dot{\phi}_{\mathrm{GVD}}=0.01$ and $-0.02\left(\Delta \tilde{\nu}_{L, 0}=73.6 \mathrm{~cm}^{-1}\right.$, see Table 1) by prism-pair adjustment. For the small experimental nonlinear refractive-index coefficient $\gamma_{2}$, the varied time chirp range covers the four performance regions from $A$ to $D$ of the SPM-GVD interaction. The experimental laser behavior in the four regions agreed with the numerical simulations described in this paper.

The small experimental SPM effect (small $\gamma_{2}$, small gain jet thickness $l_{G}$, and small absorber jet thickness $l_{A}$ ) makes the experimental prism-pair-balanced stable fundamental solitonlike pulse formation regime rather small. It may be enlarged by extending the jet thicknesses, by increasing the nonlinear refractiveindex coefficient by adding a liquid of high optical Kerr coefficient to the dye solutions, ${ }^{54}$ or by inserting an additional nonlinear sample in a third resonator cavity focus. ${ }^{55,56}$ In mode-locked solid-state lasers the SPM effect in the gain medium is larger because of its larger size.

In the GVD-balanced fundamental solitonlike laser operation regime the laser performance parameters such as pulse duration and spectral width become practically independent of the saturable absorber action after a single pulse has been formed in the self-starting mode-locking process. In this stable GVD-balanced operation regime the improved modelocking action of the saturable absorber in a collidingpulse mode-locking arrangement has no influence on the laser performance..$^{25,41,42}$

\section{Conclusions}

The influence of self-phase modulation and of groupvelocity dispersion on the operation of a passive mode-locked Rhodamine-6G-DODCI femtosecond dye laser has been analyzed numerically in detail. For a fixed frequency chirp caused by self-phase modulation, the laser behavior depends on the time chirp caused by group-velocity dispersion. For positive self-phase-modulated pulses four performance regions have been distinguished by moving from positive to negative group-velocity dispersion. These ranges are (i) the saturable-absorber-balanced positive GVD region, (ii) the periodically self-quenching region around zero GVD (some higher-order solitonlike behavior), (iii) the stable fundamental solitonlike GVD-SPM-balanced negative GVD region, and (iv) the saturable-absorber-balanced (chirp overcompensated) negative GVD region. In the case of negative self-phase-modulated pulses the same results are obtained if the sign of the GVD is changed.

In the stable fundamental solitonlike pulse formation region the saturable absorber action is needed only for background suppression in the self-starting laser process. The improved saturable absorber action in a colliding-pulse mode-locked arrangement becomes unnecessary ${ }^{25.41,42}$ (pulse duration is determined by fundamental solitonlike GVD-SPM chirp compensation).

The simulations have been carried out for the experimental parameters of a passive mode-locked Rhodamine-6G-DODCI femtosecond dye laser that operates at $620 \mathrm{~nm}$. The results obtained are quite general and give insight to passive and hybrid mode locking of dye lasers, as well as to the femtosecond pulse generation in solid-state lasers (Ti:sapphire, $\mathrm{Nd}$ :glass, and color centers).

The authors thank the Deutsche Forschungsgemeinschaft for financial support and the Rechenzentrum of the University of Regensburg for the allocation of computer time.

\section{References}

1. J. C. Diels, "Femtosecond dye lasers," in Dye Laser Principles with Applications, F. J. Duarte and L. W. Hillmann, eds. (Academic, Boston, Mass., 1990), pp. 41-132.

2. R. L. Fork, H. Avramopoulos, and J. A. Valdmanis, "Ultrashort light pulses," Am. Sci. 78, 216-223 (1990).

3. C. V. Shank, "Generation of ultrashort optical pulses," in Ultrashort Laser Pulses and Applications, W. Kaiser, ed., Vol. 60 of Topics in Applied Physics (Springer-Verlag, Berlin, 1988), pp. 5-34.

4. W. Sibbett, "Hybrid and passive mode locking in coupledcavity lasers," in Ultrafast Phenomena VII, C. B. Harris, E. P. Ippen, G. A. Mourou, and A. H. Zewail, eds., Vol. 53 of Springer Series in Chemical Physics (Springer-Verlag, Berlin, 1990), pp. 2-7.

5. C. Spielmann, F. Krausz, T. Brabec, E. Wintner, and A. J. Schmidt, "Experimental study of additive-pulse mode locking in an Nd:glass laser,' IEEE J. Quantum Electron. 27, 12071213 (1991).

6. C. Spielmann, F. Krausz, T. Brabec, E. Wintner, and A. J. Schmidt, "Femtosecond passive mode locking of a solid-state laser by a dispersively balanced nonlinear interferometer," Appl. Phys. Lett. 58, 2470-2472 (1991).

7. J. Goodberlet, J. Wang, J. G. Fujimoto, and P. A. Schulz, "Femtosecond passively mode-locked $\mathrm{Ti}: \mathrm{Al}_{2} \mathrm{O}_{3}$ laser with a nonlinear external cavity," Opt. Lett. 14, 1125-1127 (1989).

8. D. E. Spence, P. N. Kean, and W. Sibbett, "60-fsec Pulse generation from a self-mode-locked Ti:sapphire laser," Opt. Lett. 16, 42-44 (1991).

9. K. Naganuma and K. Mogi, "50-fs Pulse generation directly from a colliding-pulse mode-locked Ti:sapphire laser using an antiresonant ring mirror," Opt. Lett. 16, 738-740 (1991).

10. P. F. Curley and A. I. Ferguson, "Actively mode-locked Ti:sapphire laser producing transform-limited pulses of $150 \mathrm{fs}$ duration," Opt. Lett. 16, 1016-1018 (1991).

11. U. Keller, G. W. 'tHooft, W. H. Knox, and J. E. Cunningham, "Femtosecond pulses from a continuously self-starting passively mode-locked Ti:sapphire laser," Opt. Lett. 16, 10221024 (1991). 
12. C. Spielmann, F. Krausz, T. Brabec, E. Wintner, and A. J. Schmidt, "Femtosecond pulse generation from a synchronously pumped Ti:sapphire laser," Opt. Lett. 16, 1180-1182 (1991).

13. D. E. Spence and W. Sibbett, "Femtosecond pulse generation by a dispersion-compensated, coupled-cavity, mode-locked Ti:sapphire laser," J. Opt. Soc. Am. B 8, 2053-2060 (1991).

14. D. E. Spence, J. M. Evans, W. E. Sleat, and W. Sibbett, "Regeneratively initiated self-mode-locked Ti:sapphire laser," Opt. Lett. 16, 1762-1764 (1991).

15. A. Penzkofer, "Passive $Q$-switching and mode-locking for the generation of nanosecond to femtosecond pulses," Appl. Phys. B 46, 43-60 (1988).

16. R. L. Fork, B. I. Greene, and C. V. Shank, "Generation of pulses shorter than 0.1 ps by colliding pulse mode-locking," Appl. Phys. Lett. 38, 671-672 (1981).

17. N. Jamasbi, J. C. Diels, and L. Sarger, "Study of a linear femtosecond laser in passive and hybrid operation," J. Mod. Opt. 35, 1891-1906 (1988).

18. H. Kubota, K. Kurokawa, and M. Nakazawa, "29-fsec Pulse generation from a linear-cavity synchronously pumped dye laser," Opt. Lett. 13, 749-751 (1988).

19. L. Spinelli, B. Coulland, N. Goldblatt, and D. K. Negus, "Starting and generation of sub- 100 fs pulses in $\mathrm{Ti}: \mathrm{Al}_{2} \mathrm{O}_{3}$ by self-focusing," in Conference on Lasers and Electro-Optics, Vol. 10 of 1991 OSA Technical Digest Series (Optical Society of America, Washington, D.C., 1991), paper CPDP7.

20. G. Gabetta, H. Huang, J. Jacobson, E. P. Ippen, M. Ramaswamy, and J. G. Fujimoto, "Femtosecond puise generation in $\mathrm{Ti}: \mathrm{Al}_{2} \mathrm{O}_{3}$ using a microdot mirror mode locker," Opt. Lett. 16, 1756-1758 (1991).

21. J. Goodberlet, J. Jacobson, J. G. Fujimoto, P. A. Schulz, and F. Y. Fan, "Self-starting additive-pulse mode-locked diodepumped Nd:YAG laser," Opt. Lett. 15, 504-506 (1990).

22. G. P. A. Malcolm, P. F. Curley, and A. I. Ferguson. "Additivepulse mode locking of a diode-pumped Nd:YLF laser," Opt. Lett. 15, 1303-1305 (1990).

23. H. A. Haus, J. G. Fujimoto, and E. P. Ippen, "Structures for additive pulse mode-locking," J. Opt. Soc. Am. B 8, 2068-2076 (1991).

24. P. A. Bélanger, "Coupled-cavity mode locking: a nonlinear model," J. Opt. Soc. Am. B 8, 2077-2081 (1991).

25. J. A. Valdmanis, R. L. Fork, and J. P. Gordon, "Generation of optical pulses as short as 27 femtoseconds directly from a laser balancing self-phase modulation, group-velocity dispersion, saturable absorption, and saturable gain," Opt. Lett. 10, 131-133 (1985).

26. Y. R. Shen and G. Z. Yang, "Theory of self-phase modulation and spectral broadening," in The Supercontinuum Laser Source, R. R. Alfano, ed. (Springer-Verlag, New York, 1989), pp. 1-32.

27. G. P. Agrawal, Nonlinear Fiber Optics (Academic. Boston, Mass., 1989).

28. R. L. Fork, O. E. Martinez, and J. P. Gordon, "Negative dispersion using pairs of prisms," Opt. Lett. 9, 150-153 (1984).

29. O. E. Martinez, R. L. Fork, and J. P. Gordon, "Theory of passively mode-locked lasers including self-phase modulation and group-velocity dispersion," Opt. Lett. 9, 156-158 (1984).

30. J. C. Diels, W. Dietel, J. J. Fontaine, W. Rudolph, and B. Wilhelmi, "Analysis of a mode-locked ring laser: chirpedsolitary-pulse solutions," J. Opt. Soc. Am. B 2, 680-686 (1985).

31. J. A. Valdmanis and R. L. Fork, "Design considerations for a femtosecond pulse laser balancing self phase modulation," IEEE J. Quantum Electron. QE-22, 112-118 (1986).
32. V. Petrov, W. Rudolph, and B. Wilhelmi, "Evolution of chirped light pulses and the steady-state regime in passively modelocked femtosecond dye lasers," Rev. Phys. Appl. 22, 1639 1650 (1987).

33. H. Avramopoulos and G. H. C. New, "A numerical model for the study of phase effects in passive mode-locking," Opt. Commun. 71, 370-376 (1989).

34. H. Avramopoulos, P. M. W. French, G. H. C. New, M. A Opalinska, J. R. Taylor, and J. A. R. Williams, "Analysis of periodic pulse evolutions in a passively mode-locked ring dye laser," IEEE J. Quantum Electron. 25, 2469-2475 (1989).

35. W. L. Nighan, Jr., T. Gong, and P. M. Fauchet, "Solitons and related periodic pulse evolutions in a femtosecond ring dye laser," IEEE J. Quantum Electron. 25, 2476-2484 (1989).

36. V. Petrov, W. Rudolph, U. Stamm, and B. Wilhelmi, "Limits of ultrashort pulse generation in $\mathrm{cw}$ mode-locked dye lasers," Phys. Rev. A 40, 1474-1483 (1989).

37. H. Avramopoulos and R. L. Fork, "Bandwidth limitation and distinct operating regimes of passively mode-locked dye lasers using strong phase shaping," J. Opt. Soc. Am. B 8, 118-122 (1991).

38. A. Penzkofer and W. Bäumler, "Saturable absorption dynamics of DODCI," Opt. Quantum Electron. 23, 439-459 (1991).

39. A. Penzkofer and W. Bäumler, "Theoretical analysis of pulse development in a colliding pulse mode-locked dye laser," Opt. Quantum Electron. 23, 727-754 (1991).

40. A. Penzkofer, "Theoretical analysis of pulse shaping of selfphase modulated pulses in a grating pair compressor." Opt. Quantum Electron. 23, 685-702 (1991).

41. W. Xiang, W. Jiang, and Y. Ishida, "Femtosecond pulses generated from non-colliding pulse mode-locked ring dye lasers." Opt. Commun. 86, 70-74 (1991).

42. W. Bäumler and A. Penzkofer, "Femtosecond pulse generation in a linear passive mode-locked dye laser," Opt. Quantum Electron. 24, 313-344 (1992).

43. W. Rudolph and B. Wilhelmi, Light Pulse Compression (Harwood Academic, Chur, Switzerland, 1989).

44. A. M. Johnson and C. V. Shank. "Pulse compression in single-mode fibers-picoseconds to femtoseconds," in The Supercontinuum Laser Source, R. R. Alfano, ed. (SpringerVerlag, New York, 1989), pp. 399-449.

45. G. H. C. New, "Pulse evolution in mode-locked quasicontinuous lasers," IEEE J. Quantum Electron. QE-10, 115124 (1974).

46. F. W. Wise, I. A. Walmsley, and C. L. Tang, "Simultaneous formation of solitons and dispersive waves in a femtosecond ring laser," Opt. Lett. 13, 129-131 (1988).

47. F. Salin, P. Grangier, P. Georges, and A. Brun, "Pulse propagation near zero group-velocity dispersion in a femtosecond dye laser," Opt. Lett. 15, 1374-1376 (1990).

48. M. Wittmann, A. Penzkofer, and W. Bäumler, "Generation of frequency tunable femtosecond pulses in a cw pumped linear dispersion-balanced passive mode-locked Rhodamine 6G dye laser." Opt. Commun. 90, 182-192 (1992).

49. A. Hasegawa, Optical Solitons in Fibers, 2nd ed. (SpringerVerlag, Berlin, 1990).

50. F. Salin. P. Grangier, G. Roger, and A. Brun, "Observation of high-order solitons directly produced by a femtosecond ring laser." Phys. Rev. Lett. 56, 1132-1135 (1986).

51. F. Salin. P. Grangier, G. Roger, and A. Brun, "Experimental observation of nonsymmetrical $N=2$ solitons in a femtosecond laser," Phys. Rev. Lett. 60, 569-572 (1988).

52. F. Salin. P. Georges, and A. Brun, "Complex pulse evolution in a femtosecond laser with spectral windowing," Opt. Commun. 79, 443-447 (1990). 
53. R. S. Miranda, G. R. Jacobovitz, C. H. Brito-Cruz, and M. A. F. Scarparo, "Positive and negative chirping of laser pulses shorter than $100 \mathrm{fsec}$ in a saturable absorber," Opt. Lett. 11, 224-226 (1986).

54. M. Yamashita, K. Torizuka, and T. Sato, "Intracavity femtosecond-pulse compression with the addition of highly nonlinear organic materials," Opt. Lett. 13, 24-26 (1988).

55. C. Wang, Y. Ishida, and Y. Yamamoto, "Self-phase-modulationcontrolled passively mode-locked dye laser," Opt. Lett. 15, 965-967 (1990).

56. M. Yamashita, T. Hiraga, H. Matsuda, K. Torizuka, S. Okada, T. Moriya, and H. Nakanishi, "Femtosecond pulse compres- sion by intracavity third order nonlinear organic elements in CPM lasers," Opt. Commun. 79, 107-110 (1990).

57. W. Bäumler and A. Penzkofer, "Fluorescence spectroscopic analysis of $\mathrm{N}$ and $\mathrm{P}$ isomers of DODCI," Chem. Phys. 140, 75-97 (1990).

58. W. Bäumler and A. Penzkofer, "Accumulation of DODCI P isomers by laser excitation," Chem. Phys. 142, 431-443 (1990).

59. G. Angel, R. Gagel, and A. Laubereau, "Femtosecond polarization spectroscopy of liquid dye solutions," Chem. Phys. 131, 129-134 (1989).

60. D. Ricard and J. Ducuing, "Vibrational relaxation in the ground electronic state of large organic molecules in solution," J. Chem. Phys. 62, 3616-3619 (1975). 